\title{
Provenance of the Ediacaran Salinas Formation (Araçuaí Orogen, Brazil): Clues from lithochemical data and zircon U-Pb (SHRIMP) ages of volcanic clasts
}

\author{
Carolina Deluca $^{1 *}$ (D), Antonio Pedrosa-Soares ${ }^{1}$ (D), Sirlene Lima ${ }^{2}$ (D), Umberto Cordani3 ${ }^{3}$, Kei Sato ${ }^{3}$ (D)
}

\begin{abstract}
Salinas Formation occurs in a large region of the Northern Araçuaí orogen, Southeastern Brazil. It includes turbiditic wackes (> 10\% matrix) to arenites $(<10 \%$ matrix), pelites and clast-supported conglomerates, metamorphosed from the biotite zone of the greenschist facies to the sillimanite zone of the amphibolite facies. Salinas Formation lies unconformably on the top of or in tectonic contact with the Macaúbas Group, and hosts Cambrian granitic intrusions dated between 540 and $500 \mathrm{Ma}$. Aiming to unravel sediment provenances for the Salinas basin, we present a detailed lithochemical (45 samples) study on low-grade rocks preserved from the regional deformation, which are found in the type area of the Salinas Formation. In addition, we compare them with deformed and more metamorphic rocks of similar composition but located in other basin sectors. The lithochemical data indicate limited chemical weathering in the sediment sources, good correlations with the mineralogical compositions in respect to the variable amounts of metamorphic minerals typical of pelitic (micas, garnet, and other peraluminous silicates) and psammitic (feldspars, quartz) fractions. The main provenances of sedimentary protoliths are clearly related to continental magmatic arc and active continental margin environments. U-Pb (SHRIMP) analyses performed on zircon grains from clasts of intermediate to felsic volcanic rocks, extracted from a clast-supported metaconglomerate, yield concordant zircon $\mathrm{Pb}^{206} / \mathrm{U}^{238}$ ages from ca. $579 \mathrm{Ma}$ to ca. $697 \mathrm{Ma}$, with most of the ages in the interval of 587 to $630 \mathrm{Ma}$. This indicates that the main primary sediment source is the Rio Doce magmatic arc $(630-580 \mathrm{Ma})$, in very good agreement with the arc-related lithochemical signature. Eleven zircon crystals yield a Concordia age of $620 \pm 10 \mathrm{Ma}$, representing an important Ediacaran volcanic episode in the tectonic evolution of the Araçuaí orogen.
\end{abstract}

KEYWORDS: Lithochemistry; sediment provenance; volcanic contribution; Salinas Formation; Araçuaí orogen.

\section{INTRODUCTION}

There are useful lithochemical approaches to investigate genetic attributes and tectonic environments of metasedimentary rocks, although processes like weathering, diagenesis, and metamorphism may considerably change the composition of sedimentary materials ( $c f$. Bhatia 1985, Bhatia \& Crook 1986, Rosen 1992, McLennan et al. 1993, Slack \& Höy, 2000, Augustsson \& Bahlburg 2008, Verma \& Armstrong-Altrin 2013). The lithochemical investigation can be especially effective if combined with petrographic and isotopic studies on the preserved rocks and their modified equivalents.

In the semi-arid Jequitinhonha river valley, Northern Araçuaí orogen (Fig. 1), the Salinas Formation provides several good exposures of non-weathered metasedimentary rocks.

\footnotetext{
${ }^{1}$ Universidade Federal de Minas Gerais, Programa de Pós-Graduação em Geologia, IGC-CPMTC - Belo Horizonte (MG), Brazil. E-mails: cdelucam@gmail.com; pedrosa@cnpq.br

${ }^{2}$ Unidade de Operação da Bacia de Campos: Exploração,

Sedimentologia e Estratigrafia, Petrobras - Rio de Janeiro (RJ), Brazil.

E-mail: saalima@petrobras.com.br

${ }^{3}$ Universidade de São Paulo - São Paulo (SP), Brazil.

E-mails: ucordani@usp.br, keisato@usp.br

${ }^{*}$ Corresponding author.
}

They vary from very low-grade metasiliciclastic rocks (wacke, arenite, and pelite), which were preserved from the regional orogenic deformation, to their deformed equivalents metamorphosed up to the sillimanite zone of the amphibolite facies (Pedrosa-Soares 1995, Pedrosa-Soares \& Leonardos 1996, Pedrosa-Soares et al. 2001, 2008, Lima et al. 2002, Santos et al. 2009, Peixoto et al. 2017).

We present a detailed lithochemical investigation based on 45 samples of metasiliciclastic rocks, including 36 ones from this paper and nine compiled from Grossi-Sad and Motta (1991). Fourteen samples are from wackes metamorphosed in the very low-grade biotite zone, but preserved from orogenic deformation, collected in the type area of Salinas Formation, and 31 samples are from deformed and more metamorphic lithotypes collected in the southern sector of the Salinas basin (Fig. 1). This study is assisted by detailed petrographic examination on the analyzed lithotypes and by U-Pb (SHRIMP) analysis of zircon grains from pebbles and cobbles of intermediate to felsic volcanic rocks extracted from a Salinas metaconglomerate. The results disclose correlations between non-deformed and deformed lithotypes and reinforce the useful application of the lithochemical approach to study similar rocks in other orogenic belts. They also corroborate the orogenic nature of the Salinas basin (Lima et al.2002, Santos et al. 2009, Peixoto et al. 2015, Costa et al. 2018). This definitely links it with sediment sources in the Rio Doce magmatic arc (Tedeschi et al.2016, Novo et al. 2018). 


\section{GEOLOGICAL SETTING}

Salinas Formation is one of the most extensive units of the Northern Araçuaí orogen, which occur in large areas of the Jequitinhonha river valley (Fig. 1). Regionally, Salinas Formation covers different units of the Macaúbas Group (e.g., the Chapada Acauã and Ribeirão da Folha formations), defining a regional unconformity. It also hosts late orogenic granitic intrusions dated from ca. $540 \mathrm{Ma}$ to ca. $500 \mathrm{Ma}$ (Fig. 1).
Study of the metasedimentary rocks currently ascribed to the Salinas Formation started early in $20^{\text {th }}$ century, when they were correlated with the Macaúbas Formation (Moraes 1932). Half a century later, they were included in a separate stratigraphic unit, the Salinas Group, which was considered a more metamorphic unit intruded by granites and pegmatites that would correspond to the post-diamictite formations of Macaúbas Group (synthesis in Pedrosa-Soares 1984, and Karfunkel et al. 1985). Owing to its apparent continuity with

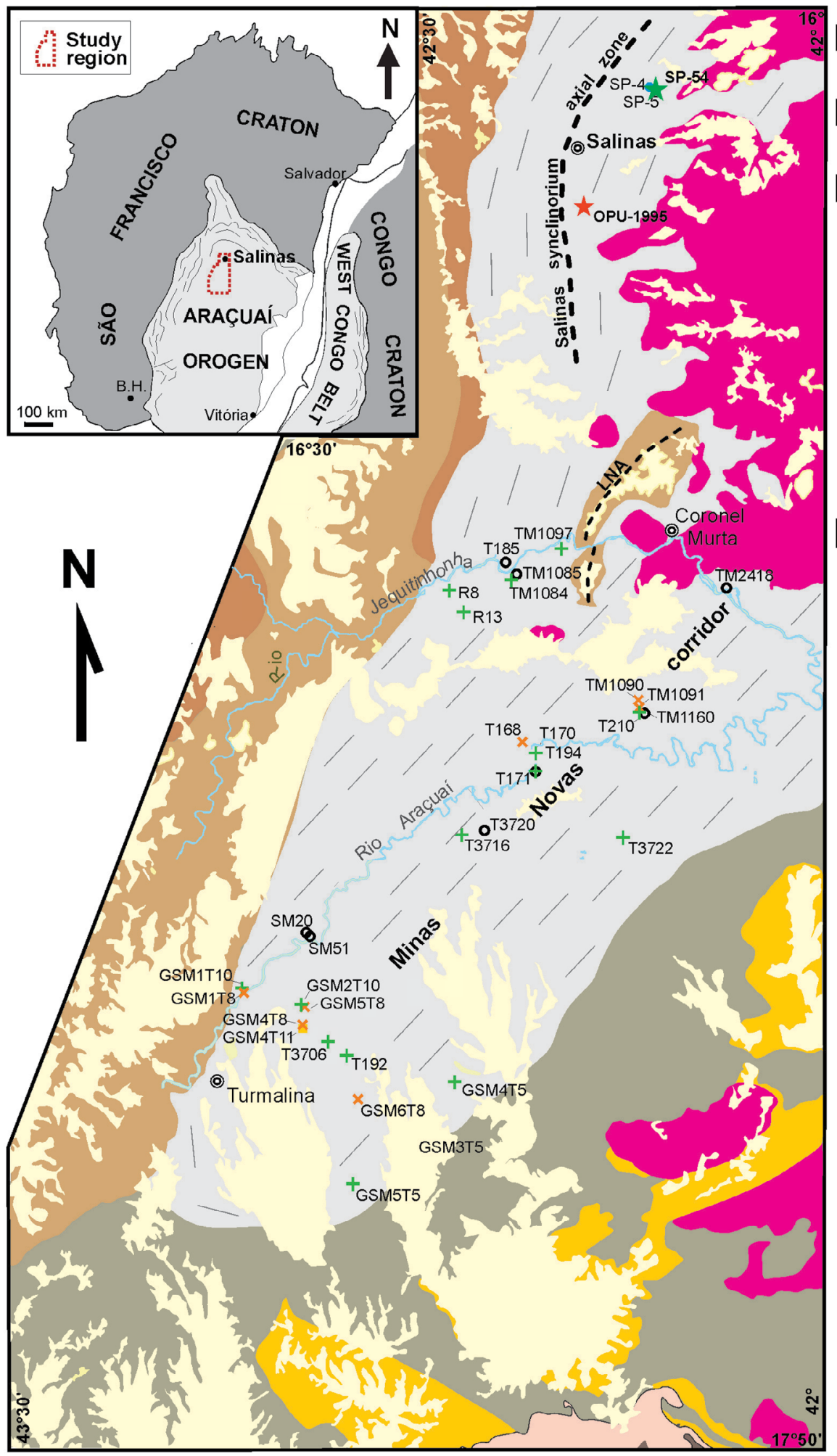

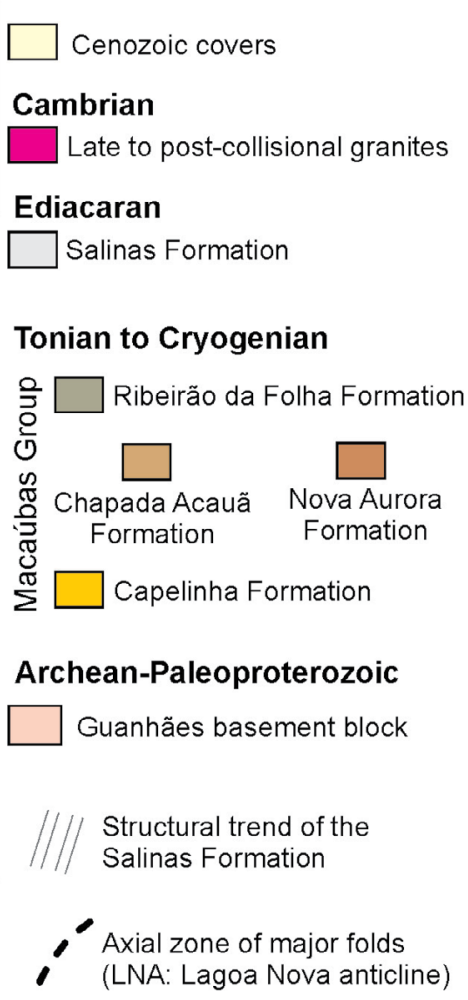

\section{Samples for lithochemical analysis}

- Salinas Formation type area

Salinas Formation in the Minas Novas corridor:

* Muscovite schist

- Carbonate schist

+ Quartz-biotite schist

- Quartzose metawacke

\section{Zircon U-Pb analysis}

+ Sample OPU-1995 (volcanic pebbles from conglomerate)

Sample SP-54 compiled U-

$\star \mathrm{Pb}$ zircon ages from Lima et al. (2002)

(-) City

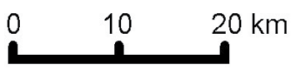

Figure 1. Location of the study region in the Araçuaí orogen shown within a paleotectonic fit including the African counterpart and a simplified geological map of the main occurrence region of the Salinas Formation (modified from Pedrosa-Soares \& Oliveira 1997; PedrosaSoares 1997a, b; Oliveira et al. 1997; Pedrosa-Soares \& Grossi-Sad 1997; Baars et al. 1997; Guimarães \& Grossi-Sad 1997). 
the regional lithofacies distribution from the proximal to distal Macaúbas Group, Salinas Formation was formerly defined as the most distal and youngest unit of Macaúbas Group (PedrosaSoares et al. 1990a, 1990b, 1992). These authors subdivided the Salinas Formation into two units: an exclusively sedimentary unit located in the proximal (Western) part of theformation, and the Ribeirão da Folha facies or member, a more distal (Eastern) metavolcanic-sedimentary succession with oceanic sediments and mafic volcanic rocks, hosting tectonic slabs of ophiolitic rocks (Pedrosa-Soares et al. 1992, 1998, 2001, 2011).

In the second half of the 1990s, the construction of the BR-251 highway linking Salinas city to the Rio-Bahia (BR116) highway led to extensive roadcuts that presented exceptional sections of the Salinas Formation completely preserved from weathering and, locally, from orogenic deformation (Pedrosa-Soares \& Oliveira 1997). These outcrops allowed detailed studies by Lima et al. (2002), which defined the main lithofacies of Salinas Formation and presented the first U-Pb geochronological data on detrital zircon grains. According to Lima et al. (2002), Salinas Formation in the type area includes laminated, banded, graded, convolute, brecciated and massive wackes, cross-bedded sandstones, metapelites (mica schists and quartz-mica schists), clast-supported conglomerates and calc-silicate rocks, which are metamorphosed from the biotite to the garnet zones of the greenschist facies. The youngest detrital zircon grains suggest a maximum sedimentation age around $568 \mathrm{Ma}$ that imply in redefinition of the Salinas Formation as a stratigraphic unit younger than the Macaúbas Group (Lima et al. 2002). Santos et al. (2009) presented detailed tectonic studies on the Northern Salinas Formation, which showed its progressive deformation from completely non-deformed to tightly folded and transposed zones, and suggested a model of flysch-type basin evolving from the pre-collisional to collisional stages of the Araçuaí orogen. Costa et al. (2018), based on the lithofacies investigation in the type area of Salinas Formation, characterized the Salinas basin as a large and curved trough, open to South-Southwest. According to these authors, accumulation in the Salinas Basin occurred along its axis, with general Southwest-South progradation of a turbidite fan system fed from the North. The detailed metamorphic studies presented by Peixoto et al. (2018) demonstrate a double metamorphic regime along the Salinas synclinorium (Fig. 1), with a Barrovian-type event related to the collisional tectonics and a Buchan-type event related to the plutonic igneous activity associated with the gravitational collapse of Araçuaí orogen. Mineral assemblages of metapelites in the Salinas synclinorium indicate maximum metamorphic conditions around $640^{\circ} \mathrm{C}$ at $5.5 \mathrm{kbar}$, with no piece of evidence of partial melting (Peixoto et al. 2018).

To the South of Salinas synclinorium, the typical rock assemblage of Salinas Formation continues to occur along the Minas Novas corridor (Pedrosa-Soares et al. 1993, PedrosaSoares 1995, Alkmim et al. 2006), a double-verging transpressive structure extending from Turmalina - Minas Novas to Coronel Murta - Araçuaí regions (Fig. 1). In contrast to the well-preserved metasedimentary rocks from orogenic deformation locally found in the type area of the Salinas Formation (e.g., outcrops SP-4 and SP-5, Fig. 1), no rock exposure free from the regional deformation has been found in the Minas Novas corridor yet. The Salinas lithotypes in Minas Novas corridor are tightly folded quartz-biotite schists, quartzose metawackes (also called "impure quartzites"), carbonate schists, muscovite schists, calc-silicate rocks and sparse clast-supported metaconglomerate lenses, which frequently show the sedimentary layering tectonically transposed by the regional foliation (Pedrosa-Soares 1995). Along the Minas Novas corridor, Al-rich schists of Salinas Formation display intermediate to low pressure regional metamorphism with increasing temperature from SW to NE, passing through the biotite, garnet, staurolite, andalusite, and cordierite zones (Pedrosa-Soares et al. 1993, Pedrosa-Soares \& Leonardos 1996).

\section{ANALYTICAL METHODS}

Besides conventional field and petrographic studies, this paper presents data from lithochemical analysis and $\mathrm{U}-\mathrm{Pb}$ (SHRIMP) dating of zircon grains.

\section{Lithochemical analysis}

The selected representative samples are free of weathering and hydrothermal alteration, with no veins or fractures filled by secondary minerals. The samples were firstly cleaned and prepared using the conventional methods (crushing and milling) in the laboratories of the Centro de Pesquisas Professor Manoel Teixeira da Costa (CPMTC), Universidade Federal de Minas Gerais, and Department of Geology, Universidade Federal de Ouro Preto. Major oxides and trace elements including rare-earth elements (REE) were determined through the Inductively Coupled Plasma Mass Spectrometry (ICP-MS) on 14 whole-rock samples of low-grade (biotite zone) metawackes from the type area of Salinas Formation (outcrops SP-04 and SP-05, Fig. 1). Analyses were performed by the Actlabs (Activation Laboratories), in Canada. The ICP analysis needs complete dissolution, which is possible by melting the powdered rock with lithium tetraborate $\left(\mathrm{Li}_{2} \mathrm{~B}_{4} \mathrm{O}_{7}\right)$ firstly with subsequent attack by multi-acid solution $\left(\mathrm{HCl}, \mathrm{HNO}_{3}, \mathrm{HF}\right.$ and $\left.\mathrm{HClO}_{4}\right)$. Determinations of $\mathrm{FeO}$ and $\mathrm{Fe}_{2} \mathrm{O}_{3}$ were done using colorimetric and volumetric methods at the CPMTC-UFMG.

Thirty-one whole-rock analysis (major and trace elements) correspond to samples of clastic metasedimentary rocks of Salinas Formation collected in the Minas Novas corridor (Fig. 1). Twenty-two analyses are unpublished and the other nine were compiled from Grossi-Sad and Motta (1991). These rocks include quartz-biotite schist, muscovite schist, carbonate schist and quartzose metawacke, displaying regional metamorphism from garnet to staurolite zones (Fig. 1). Those 31 samples were analyzed in the GEOSOL Laboratory in Brazil by atomic absorption spectrometry, X-ray fluorescence spectrometry, ICP atomic emission spectrometry (ICP-AES), colorimetric and volumetric methods. Samples were melted with lithium tetraborate to determine major elements through the X-ray fluorescence. In order to analyze trace elements, including REE, by ICP-AES, the samples were melted with lithium metaborate and decomposed using a multi-acid solution $\left(\mathrm{HCl}, \mathrm{HNO}_{3}, \mathrm{HF}\right.$ and $\left.\mathrm{HClO}_{4}\right)$. 
Whole-rock classification diagrams and molar element ratios were obtained using the GCDkit 2.3 software (Janousek et al. 2006).

\section{$\mathrm{U}-\mathrm{Pb}$ (SHRIMP) analysis}

The U-Pb analysis of OPU-1995 sample was performed on zircon grains using the Sensitive High-Resolution Ion Microprobe (SHRIMP II) of the Chinese Academy of Geological Sciences, by means of the procedure described by Williams (1998). The analyses were made by UGC, at the São Paulo laboratory, operating the Chinese facilities through remote access, via the internet. The zircon grains were concentrated from pebbles and cobbles of volcanic rocks extracted from a clast-supported metaconglomerate of Salinas Formation. The separated volcanic clasts were cleaned as much as possible, although it was not possible to completely remove all traces of the matrix. Zircon grains were separated using the conventional methods (crushing, grinding, gravimetric and magnetic-Frantz isodynamic separator) and handpicked under binocular microscope at the LOPAG laboratory of the Universidade de Ouro Preto, Brazil. After mounted in epoxy resin and polished to expose their centers, cathodoluminescence $(C L)$ images were prepared for all grains to identify their morphological features and internal structures.

To better understand significant tectonic processes evolving the Salinas Basin and to track its sediment sources, we also compiled and re-calculated the zircon U-Pb (SHRIMP) raw data from the wacke sample (SP-54) of the Salinas Formation type area formerly presented by Lima et al. (2002). This procedure improved the presentation quality of the original data, incorporating more spots than those previously considered. The Concordia diagrams and probability density plots were prepared with Isoplot/Ex (Ludwig 2003).

\section{RESULTS AND INTERPRETATION}

We synthesize field, petrographic, geochronological and lithochemical studies, comparing the results from the samples collected in the type area of Salinas Formation, located in the surroundings of Salinas city, with the samples collected along the Minas Novas corridor (Fig. 1).

\section{Petrography}

The best well-preserved exposures of Salinas Formation are located in the type area (Fig. 1), where successions of metawackes (matrix $>10 \%$ ) to meta-arenites (matrix $<10 \%$ ), metapelites and clast-supported metaconglomerates (meta-orthoconglomerates) are found. Compositionally, metawackes and meta-arenites only differ by the amount of clay-related matrix represented by mica content, reflecting small differences in rock tints. Although metamorphosed to greenschist facies, those rocks can be found completely unaffected by the orogenic deformation (Lima et al. 2002, Costa et al.2018), as well as showing tectonic structures imprinted by the progressive deformation related to the collisional stage of Araçuaí orogen (Santos et al.2009, Peixoto et al.2018). Successions preserved from the orogenic deformation include massive to graded metawackes, banded to laminated metawackes, convolute metawackes, and cataclastic to brecciated metawackes, with sedimentary structures and striking evidence of syn-sedimentary tectonic activity (Fig. 2).

The massive metawackes are generally medium-tofine grained light-grey rocks that show a granoblastic to grano-lepidoblastic texture with weak recrystallization. They contain quartz, biotite, plagioclase, white mica, calcite, K-feldspar, and lithic fragments in variable proportions. Tourmaline, garnet, apatite, zircon, titanite and opaque minerals are accessories. Graded bedding is materialized by variation in the biotite amount representing the mud fraction, or by textural gradation of quartz-feldspar-rich matrix (Fig. 2). Upwards-coarsening graded bedding is a common feature in metawackes. The banded to laminated metawackes consist of bands and laminae relatively rich in biotite alternated with those of graded to massive metawackes (Fig. 2). The biotite-rich bands and laminae vary in composition from pelite-rich metawacke (biotite-rich metasandstone) to metapelite (quartz-mica schist to mica schist). The pelitic schists mark the regional metamorphism and may reach the garnet zone in the Salinas Formation type area.

Metric to decametric lenses of polymictic clast-supported metaconglomerates (orthoconglomerates) occur near the top of Salinas Formation (Fig. 3); therefore, they characterize a general upwards-coarsening succession (Santos et al. 2009). The scarce matrix has wacke composition similar to the metawacke layers hosting the orthoconglomerate lenses (Fig. 3A). Clasts are generally well-rounded and can be stretched and rotated along the regional ductile foliation. They are pebbles and cobbles of volcanic and subvolcanic rocks together with clasts of quartz, quartzite, gneiss, granite, and carbonate rock. Most clasts of volcanic rocks vary in composition from andesite to rhyolite, being generally porphyritic with feldspar phenocrysts immersed in fine-grained matrix (Figs. 3B to 3D). The Salinas metaconglomerate lenses, sparsely occurring from the type area southwards, roughly follow the axial zone of the Salinas synclinorium and continue along the central zone of Minas Novas corridor (where they mainly occur in the Araçuaí River banks and subsidiary creeks).

From the type area, located in the axial zone of Salinas synclinorium, to the South and East, the Salinas Formation tends to become more metamorphic and deformed by the orogenic events that formed the Araçuaí orogen. This is the case of rocks found in Minas Novas corridor, from Coronel Murta to Turmalina (Fig. 1), in which the formation comprises successions of quartz-mica schist rich in biotite, muscovite schist, carbonate schist and quartzose metawacke, with lenses of calc-silicate rocks and clast-supported metaconglomerates (Figs. 1 and 4). These rocks show, at least, the penetrative regional foliation and prominent mineral lineation (Fig. 4). Generally, they are tightly folded and may show the sedimentary bedding transposed along fold hinges (Fig. 4C). The alternating bands and laminae with variably amounts of quartz, biotite and feldspars resemble the banded to laminated metawackes of the type area, and locally graded bedding and water-escape structures are preserved. Besides some metamorphic minerals not found in the type area (e.g., staurolite, cordierite), the mineralogical 
compositions of the psammitic to pelitic rocks along the Minas Novas corridor are similar to the non-deformed and weakly deformed lithotypes of Salinas Formation.

These rocks are essentially composed of variably contents of quartz, biotite, muscovite, and plagioclase. Calcite, K-feldspar, garnet, apatite, zircon, titanite, tourmaline, monazite, and opaque minerals are accessory phases. The carbonate schist is rich in quartz and biotite, with a significant amount of calcite. The muscovite schist is poor in Fe-rich minerals, which probably reflects a non-oxidizing environment during the sedimentation. Therefore, the banded to laminated packages composed of quartzose metawacke, carbonate schist and quartz-mica schist correspond to the successions of massive to graded, banded to laminated metawackes with intercalations of more pelitic terms.
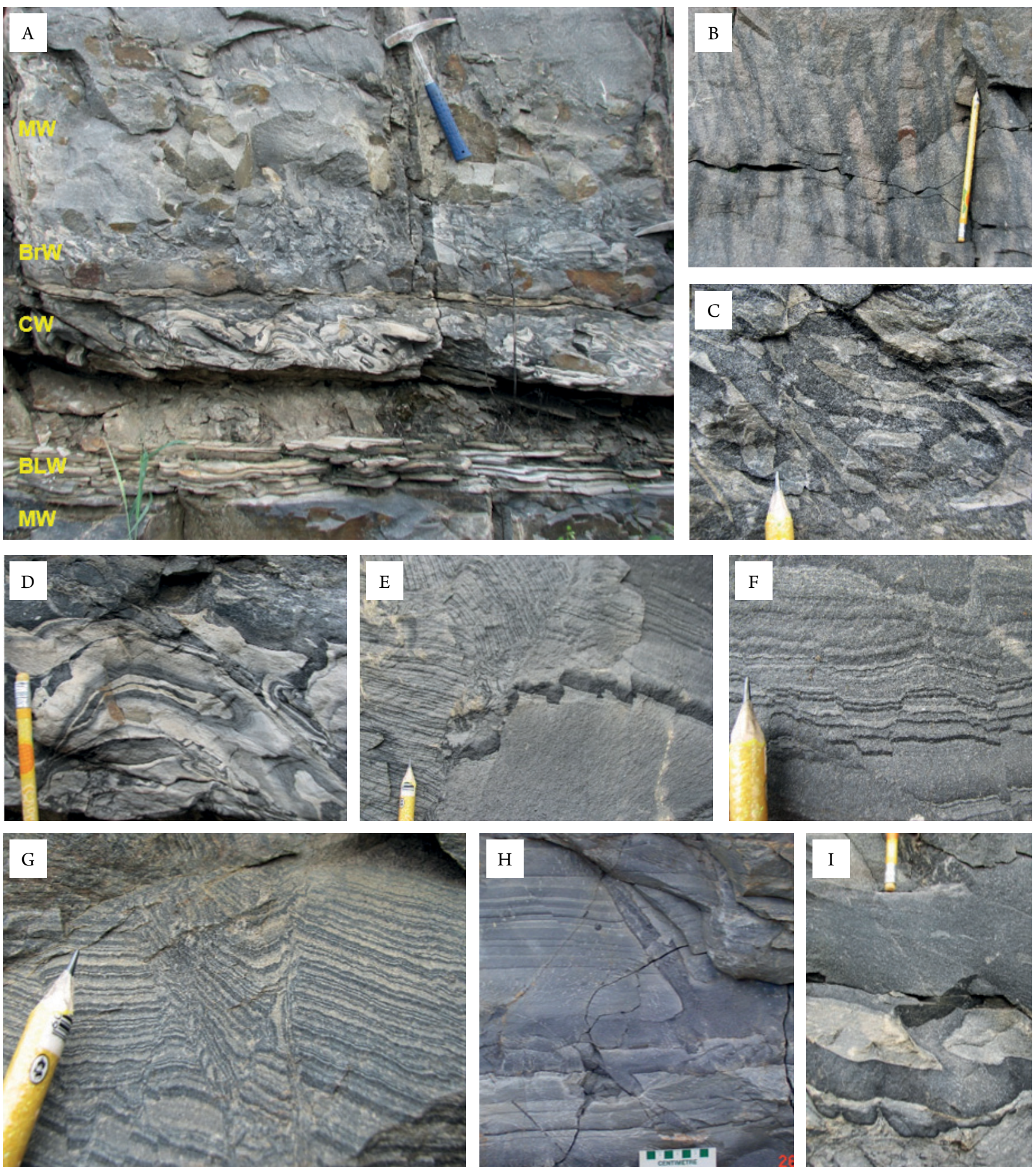

Figure 2. The Salinas Formation in outcrops of the type area (UTM: 801065 - 8222433; BR-251 highway roadcuts, $24 \mathrm{~km}$ to the east from the junction with MG-404 road), showing rocks without orogenic deformation but metamorphosed in the biotite zone of the greenschist facies: A, succession of banded to laminated wacke (BLW), convolute wacke (CW) and brecciated wacke (BrW), between massive wacke (MW) layers; B, water-escape structure marked by biotite-rich (i.e., mud-rich) darker upright flames in massive wacke; C, detail of brecciated wacke; D, detail of convolute wacke; E, cataclastic laminated wacke filling space in a syn-sedimentary extensional (growth) fault cutting massive wacke; F, syn-sedimentary (growth) micro-faults and graben-horst micro-pattern in laminated to banded wacke with coarsening-up graded layering done by the gradual decreasing of biotite content from the base (darker) to top (lighter); G, syn-sedimentary fault with drag microfolds in laminated wacke; $\mathrm{H}$, water-escape structures along syn-sedimentary faults, cutting across coarsening-up graded, banded to laminated wackes; I, flame structures outlined by biotite-rich (i.e., mud-rich) bands, covered by massive wacke. 
Regionally, field and petrographic features of the main lithofacies associations indicate that Salinas Formation mostly comprises a turbiditic deep-sea sand-mud sequence, deposited in a basin episodically affected by syn-sedimentary tectonic activity, characterizing a flysch-type orogenic basin, as already suggested by other authors (Lima et al. 2002, Santos et al. 2009, Peixoto et al. 2015).

\section{Lithochemistry}

Results of major and trace element analysis for the wholerock samples of Salinas Formation are listed in Tables 1, 2 (major element oxides) and 3 (trace elements). We will firstly consider the potential influences of sedimentary processes on lithochemical data and, then, evaluate the data in terms of protolith and depositional environment interpretations.

\section{Data evaluation and protolith interpretation}

The chemical composition of sediments is a complex system influenced by the petrological evolution of the source rocks, as well as sedimentary maturation, weathering and diagenesis. Although diagenesis can promote significant changes in the chemical composition of siliciclastic sediments, lithochemical data from sedimentary and metasedimentary rocks may be useful to interpret their origin. Even if clastic fractions (e.g., feldspars and lithoclasts) are selectively dissolved or replaced by authigenic minerals during diagenesis (Morton \& Hallsworth 1999), the overall lithochemical signature can be preserved. Erosion and transport of weathered rocks may produce chemical differentiation, but not chemical changes, through the selection of transported sediments (Nesbitt \& Young 1984). Despite the variable intensity of these processes, weathering is the main cause of compositional change in siliciclastic rocks (Nesbitt 2003). According to Nesbitt and Young (1984), the weathering degree of the source rock can be measured through the chemical index of alteration $\left(\mathrm{CIA}=\left[\mathrm{Al}_{2} \mathrm{O}_{3} /\left(\mathrm{Al}_{2} \mathrm{O}_{3}+\mathrm{CaO}^{*}+\mathrm{Na}_{2} \mathrm{O}\right.\right.\right.$ $\left.\left.\left.+\mathrm{K}_{2} \mathrm{O}\right)\right] \times 100\right)$ ). The $\mathrm{SiO}_{2} / \mathrm{Al}_{2} \mathrm{O}_{3}$ ratio can be useful to indicate the degree of sediment maturity, reflecting the increase of the quartz fraction in relation to clay fraction during transport and recycling of sediments (Roser \& Korsch 1999). The $\mathrm{K}_{2} \mathrm{O} /$ $\mathrm{Na}_{2} \mathrm{O}$ ratio indicates the proportion of potassic phases (e.g., $\mathrm{K}$-feldspar, common micas, illite) in relation to plagioclase, and the $\mathrm{MgO} / \mathrm{CaO}$ ratio can represent the relative contribution of iron-magnesian minerals and plagioclase in carbonate-free rocks (Pedrosa-Soares 1995, and references therein).
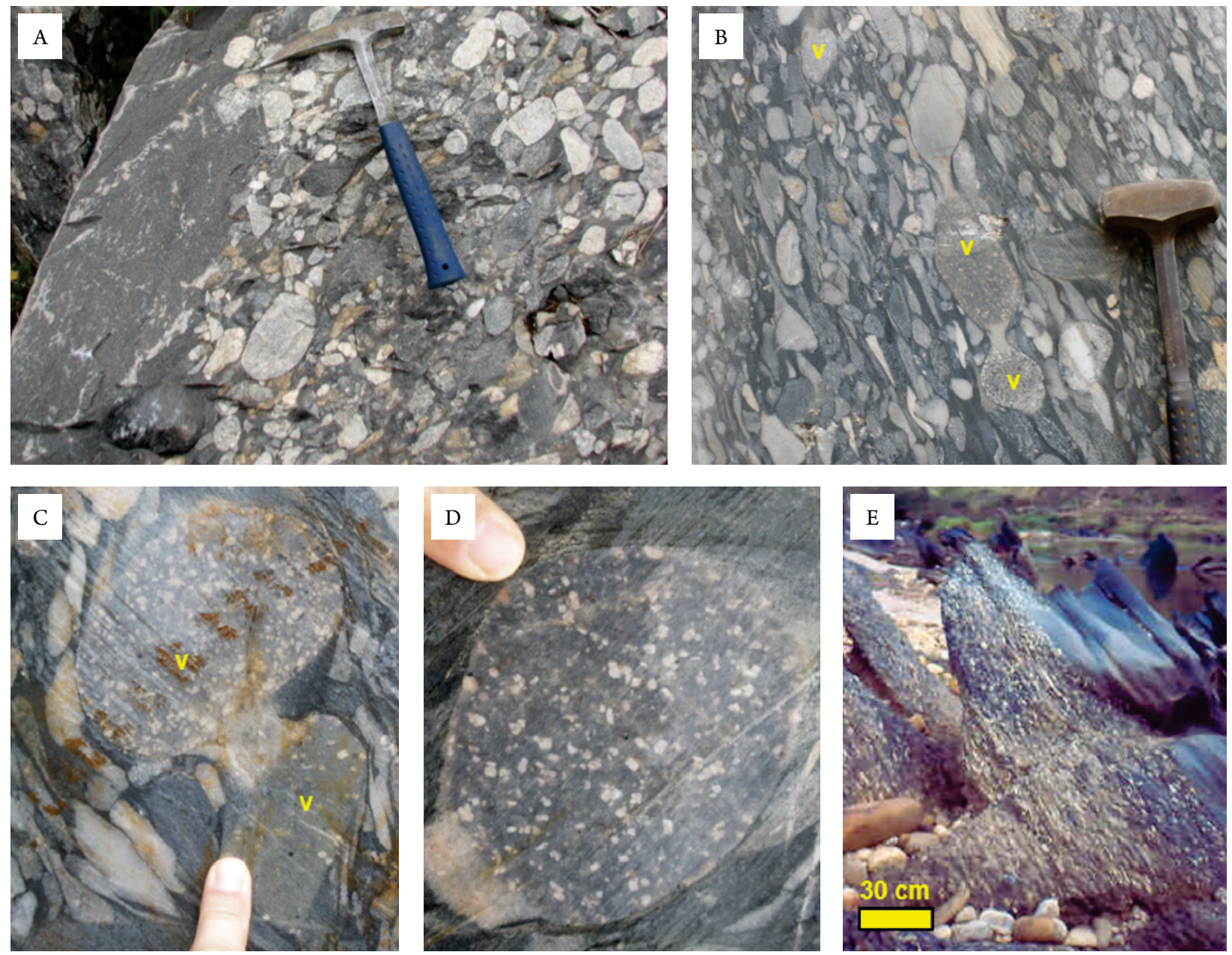

Figure 3. Clast-supported metaconglomerate (meta-orthoconglomerate) lenses of the Salinas Formation: A, outcrop in a quarry for dimension stone, showing the contact of a conglomerate lens and massive wacke (UTM: 789473 - 8220347; road MG-404, $12 \mathrm{~km}$ to the north of the road junction with the BR-251 highway); B, detail of the same metaconglomerate, showing stretched and rotate clasts (v, volcanic rocks); C, porphyritic volcanic rocks (v) of intermediate to felsic composition; D, pebble of porphyritic dacite; E, lens of clast-supported metaconglomerate intercalated with quartz-mica schist in the Minas Novas corridor (left bank of the Araçuaí River in front of the mouth of the Fanado River). 
The individual chemical index of alteration (CIA) values for Salinas samples are low to intermediate, between 47 and 69 (Fig. 5), with a general average of 58, which is very close to the average CIA $(=50)$ for the non-weathered upper crust (Taylor \& McLennan 1985). The distinct lithotypes of Salinas Formation show the following CIA averages: metawackes of the type area, 54; carbonate schists, 54; quartzose metawacke, 52; quartz-biotite schist, 62; and muscovite schist, 64 . The general CIA average for the samples from Minas Novas corridor is 59, a little bit greater than the average CIA for the type area (54). In terms of lithotype correlations, the CIA values of the metawackes from the type area (54) and carbonate schist (54) are virtually equal, and a little higher than the CIA (52) of the quartzose metawacke, indicating a solid correlation between these lithotypes relatively rich in immature sand fraction. Indeed, the higher the mica content the greater the CIA value, as shown by the quartz mica schist (average CIA =62) and muscovite schist (average CIA = 64), which indicate an increase of clay fraction and, consequently, more weathered sources.

The $\mathrm{Al}_{2} \mathrm{O}_{3}-\left(\mathrm{CaO}^{*}+\mathrm{Na}_{2} \mathrm{O}\right)-\mathrm{K}_{2} \mathrm{O}$ diagram (Fig. 5) confirms the feebly weathered nature for the sedimentary protoliths, showing a moderate tendency toward the $\mathrm{Al}_{2} \mathrm{O}_{3}$ vertex, which corresponds to the maximum CIA. The Salinas samples outline
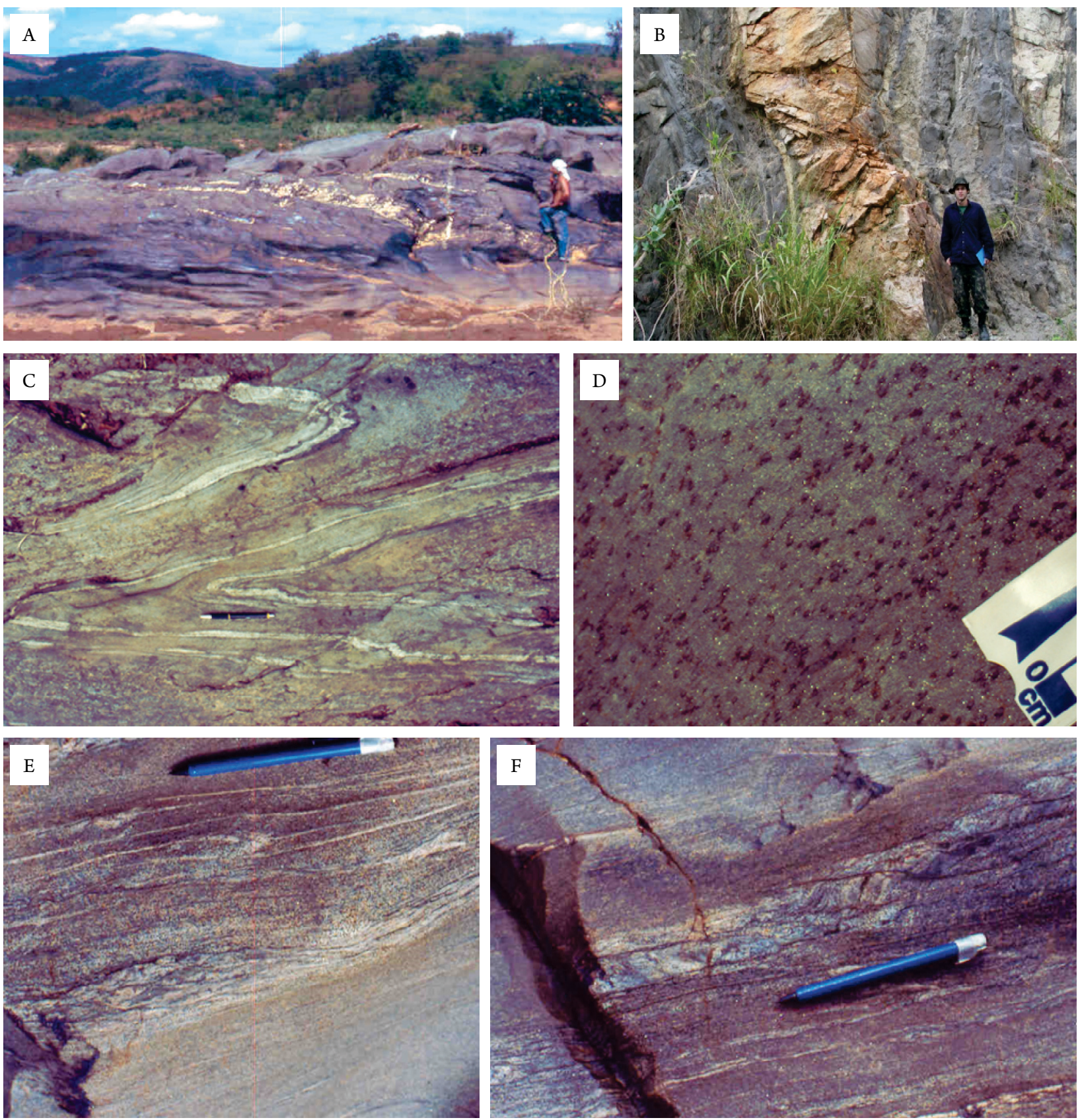

Figure 4. Outcrops of deformed and metamorphosed rocks of the Salinas Formation in the Minas Novas corridor: A, despite the rock composition, outcrops generally are dark gray to black owing to coats rich in manganese oxide (the so-called desert varnish); photo A also shows quartz veins, locally rich in gold, host by quartz-mica schist and metawacke (see Pedrosa-Soares \& Leonardos, 1996); B, quartz-mica schist hosting pegmatite veins (close to Coronel Murta); C, folded banded quartz-mica schist with concordant quartz veins (close to Virgem da Lapa); D, muscovite schist with biotite and garnet porphyroblasts (close to Minas Novas); E, banded to laminated metawacke; F, carbonate schist (dark grey upper part), quartzose metawacke (light gray middle part) and laminated metawacke (lower part). The outcrops A, E and F are located in the Funil area, Araçuaí River banks, south of Virgem da Lapa. 
Braz. J. Geol. (2019), 49(2): e20190017

Table 1. Major element (wt\%) compositions of clastic metasedimentary rocks from the Salinas Formation.

\begin{tabular}{|c|c|c|c|c|c|c|c|c|c|c|c|c|c|c|}
\hline Sample & Lithofacies & $\mathrm{SiO}_{2}$ & $\mathrm{TiO}_{2}$ & $\mathrm{Al}_{2} \mathrm{O}_{3}$ & $\mathrm{Fe}_{2} \mathrm{O}_{3}$ & $\mathrm{FeO}$ & FeOt & MnO & MgO & $\mathrm{CaO}$ & $\mathrm{Na}_{2} \mathrm{O}$ & $\mathrm{K}_{2} \mathrm{O}$ & $\mathbf{P}_{2} \mathbf{O}_{5}$ & LOI \\
\hline SM51 & & 46.9 & 1,00 & 20.4 & 3.3 & 5.1 & 8.07 & 0.1 & 3.3 & 4.9 & 1.3 & 6.9 & 0.19 & 6.19 \\
\hline $\mathrm{T} 171 \mathrm{C}$ & & 58.7 & 0.79 & 14.9 & 1.6 & 4.8 & 6.24 & 0.29 & 3.5 & 6.4 & 1.6 & 2.7 & 0.14 & 4.28 \\
\hline T3720 & & 65.2 & 0.92 & 12.9 & 1.6 & 2.8 & 4.24 & 0.55 & 3.7 & 4.4 & 2.9 & 1.9 & 0.29 & 2.57 \\
\hline SM20 & Carbonate & 66.7 & 0.73 & 11.1 & 0.72 & 3.4 & 4.04 & 0.09 & 1.8 & 4.9 & 2.8 & 2.3 & 0.2 & 5.12 \\
\hline TM1085 & schist & 68.3 & 0.81 & 13.1 & 1,00 & 3.32 & 4.22 & 0.11 & 1.6 & 3,00 & 2.8 & 2.5 & 0.18 & 1.73 \\
\hline TM1160 & & 68.6 & 0.74 & 12,00 & 1.5 & 2.89 & 4.24 & 0.11 & 1.6 & 3.8 & 2.7 & 2.2 & 0.17 & 1.92 \\
\hline $\mathrm{T} 185$ & & 69.8 & 0.64 & 13,00 & 0.48 & 2.9 & 3.33 & 0.09 & 1.8 & 3.1 & 2.3 & 2.4 & 0.17 & 3.12 \\
\hline TM2418 & & 72.1 & 0.56 & 11.4 & 1.5 & 2.16 & 3.51 & 0.13 & 1.5 & 3.2 & 1.4 & 2.6 & 0.15 & 1.14 \\
\hline SP4A & & 70.54 & 0.66 & 12.73 & 0.98 & 4.24 & 5.12 & 0.23 & 1.12 & 1.25 & 3.74 & 3.22 & 0.2 & 0.93 \\
\hline SP4B & & 65.12 & 0.75 & 15.93 & 0.94 & 4.41 & 5.26 & 0.1 & 2.19 & 0.85 & 3.73 & 4.67 & 0.2 & 0.86 \\
\hline SP4C & & 62.39 & 0.79 & 16.81 & 1.26 & 4.48 & 5.61 & 0.14 & 2.73 & 1.52 & 3.15 & 5.18 & 0.19 & 0.8 \\
\hline SP4D & & 76.81 & 0.49 & 11.93 & 1.10 & 1.73 & 2.72 & 0.12 & 0.7 & 0.67 & 3.7 & 1.55 & 0.13 & 0.91 \\
\hline SP4E & & 70.01 & 0.59 & 14.66 & 1.10 & 2.83 & 3.82 & 0.08 & 1.55 & 0.58 & 3.5 & 3.99 & 0.18 & 1,00 \\
\hline SP4F & Salinas & 74.43 & 0.59 & 12.08 & 1.56 & 2.20 & 3.60 & 0.07 & 0.77 & 0.57 & 3.43 & 3.27 & 0.14 & 0.8 \\
\hline SP4G & Type area & 70.47 & 0.52 & 14.51 & 0.45 & 3.03 & 3,00 & 0.13 & 1.22 & 0.92 & 3.64 & 4.09 & 0.16 & 0.66 \\
\hline SP4H & (metawacke) & 72.26 & 0.48 & 12.77 & 0.73 & 3.34 & 4,00 & 0.2 & 1.05 & 0.87 & 4.25 & 3.3 & 0.14 & 0.33 \\
\hline SP4I & & 63.82 & 0.8 & 16.71 & 1.08 & 4.50 & 5.57 & 0.11 & 2.39 & 1.01 & 2.41 & 5.39 & 0.21 & 1.17 \\
\hline SP4J & & 73.8 & 0.5 & 11.74 & 0.31 & 3.73 & 4.01 & 0.27 & 0.8 & 1.89 & 3.68 & 1.8 & 0.13 & 1.03 \\
\hline SP5A & & 65.46 & 0.56 & 15.5 & 0.05 & 3.53 & 3.57 & 0.15 & 1.68 & 2.04 & 4.57 & 3.61 & 0.13 & 1.2 \\
\hline SP5B & & 64.91 & 0.66 & 14.74 & 1.09 & 4.68 & 5.66 & 0.13 & 2.36 & 1.56 & 2.3 & 5.9 & 0.22 & 0.96 \\
\hline SP5C & & 72.34 & 0.39 & 14.11 & - & - & 2.96 & 0.05 & 1,00 & 1.21 & 5.68 & 1.47 & 0.16 & 0.65 \\
\hline SP5D & & 66.15 & 0.56 & 15.66 & - & - & 3.94 & 0.09 & 1.67 & 2.07 & 4.66 & 3.68 & 0.17 & 1.14 \\
\hline GSM1T8* & & 59.3 & 0.74 & 19,00 & 5.4 & 2.1 & 6.95 & 0.26 & 3,00 & 1,00 & 1.3 & 5.2 & 0.15 & 1.78 \\
\hline GSM4T8* & & 63.8 & 0.92 & 16.6 & 4.7 & 2.4 & 6.63 & 0.11 & 2.8 & 0.43 & 1.9 & 3.8 & 0.19 & 1.95 \\
\hline GSM5T8* & & 64.7 & 0.93 & 16.6 & 3.9 & 2.8 & 6.31 & 0.18 & 2.6 & 0.4 & 2,00 & 3.7 & 0.19 & 1.56 \\
\hline GSM6T8* & Muscovite & 65,0 & 0.8 & 16.4 & 4.5 & 2.2 & 6.25 & 0.1 & 2.6 & 0.29 & 1.9 & 3.5 & 0.16 & 1.85 \\
\hline T171B & schist & 57.3 & 0.89 & 18.6 & 4.6 & 3.5 & 7.64 & 0.1 & 3.8 & 1.6 & 2.3 & 5,00 & 0.15 & 1.96 \\
\hline T168 & & 58.1 & 0.81 & 18.8 & 4.2 & 3.5 & 6.81 & 0.26 & 3.8 & 1.8 & 1.4 & 5.1 & 0.15 & 1.9 \\
\hline TM1091 & & 58.9 & 0.89 & 17.4 & 4.2 & 3.03 & 6.98 & 0.59 & 3.3 & 2.3 & 2.9 & 4.1 & 0.2 & 0.81 \\
\hline GSM3T11* & Quartzose & 77.8 & 0.65 & 9.8 & 0.82 & 2.5 & 3.24 & 0.09 & 1.3 & 1.3 & 3.2 & 1.3 & 0.21 & 0.79 \\
\hline GSM4T11* & metawacke & 77.8 & 0.62 & 9.8 & 1.2 & 2.2 & 3.28 & 0.06 & 1.1 & 1,00 & 3.7 & 1.5 & 0.21 & 0.43 \\
\hline GSM2T10* & & 70.3 & 0.76 & 13.5 & 2.9 & 2.7 & 5.31 & 0.13 & 1.1 & 1.6 & 2.9 & 2.4 & 0.19 & 1.07 \\
\hline GSM4T5* & & 63.3 & 1.1 & 16.4 & 2.1 & 4.9 & 6.79 & 0.16 & 2.8 & 0.63 & 2.2 & 4.1 & 0.17 & 1.83 \\
\hline GSM5T5* & & 65.1 & 0.89 & 16.1 & 1.4 & 5,00 & 6.26 & 0.11 & 2.7 & 0.53 & 2.2 & 3.3 & 0.17 & 2.06 \\
\hline R13 & & 58.7 & 0.78 & 18.3 & 2.2 & 5,00 & 6.76 & 0.35 & 3.2 & 2.4 & 2,00 & 4.6 & 0.15 & 2.04 \\
\hline TM1084 & & 60,0 & 0.83 & 17.7 & 1.9 & 5.05 & 5.77 & 0.37 & 2.6 & 2.3 & 2.8 & 4.4 & 0.18 & 0.83 \\
\hline T3721 & Quartz- & 61.5 & 0.79 & 16.6 & 1.3 & 4.6 & 6.78 & 0.41 & 3.5 & 3.5 & 3.4 & 2.7 & 0.25 & 1.13 \\
\hline T192A & biotite & 61.9 & 0.92 & 17.8 & 2.2 & 4.8 & 6.9 & 0.14 & 2.9 & 0.57 & 2.2 & 3.9 & 0.16 & 2.15 \\
\hline $\mathrm{T} 210$ & schist & 62.0 & 0.86 & 16.9 & 3,00 & 4.2 & 6.25 & 0.15 & 3.6 & 1.4 & 2.3 & 3.7 & 0.17 & 1.5 \\
\hline $\mathrm{T} 170 \mathrm{C}$ & & 62.0 & 0.86 & 16.9 & 2.5 & 4,00 & 6.67 & 0.05 & 2.9 & 2.6 & 1.8 & 4.7 & 0.18 & 1.28 \\
\hline TM1097 & & 64.1 & 1,00 & 15.1 & 1.8 & 5.05 & 5.85 & 0.16 & 2.6 & 1.2 & 2.8 & 3.6 & 0.21 & 0.43 \\
\hline T3706 & & 64.1 & 0.98 & 15.8 & 0.72 & 5.2 & 5.11 & 0.09 & 4.4 & 0.47 & 2.3 & 3.4 & 0.18 & 1.11 \\
\hline $\mathrm{R} 8 \mathrm{~B}$ & & 64.9 & 0.73 & 16.1 & 1.9 & 3.4 & 5.94 & 0.09 & 2.5 & 2.1 & 2.4 & 4.1 & 0.19 & 1.31 \\
\hline T3722 & & 66.3 & 0.91 & 15.3 & 0.82 & 5.2 & 5.67 & 0.1 & 3.3 & 0.71 & 1.9 & 3.2 & 0.2 & 1.64 \\
\hline T3716 & & 66.8 & 0.73 & 14.2 & 0.97 & 4.8 & 5.67 & 0.08 & 4.6 & 0.41 & 1.2 & 4,00 & 0.15 & 1.91 \\
\hline UCC & & 66.0 & 0.5 & 15.2 & - & - & 4.5 & 0.1 & 2.2 & 4.2 & 3.9 & 3.4 & 0.2 & - \\
\hline
\end{tabular}

${ }^{*}$ Grossi-Sad \& Motta (1991); and UCC (Upper continental crust average, Taylor \& McLennan 1985). 
Table 2. Chemical values calculated from major elements composition of Salinas metasedimentary rocks.

\begin{tabular}{|c|c|c|c|c|c|c|c|c|c|c|c|c|c|c|c|c|c|c|c|c|c|}
\hline \multirow{2}{*}{ Sample } & \multirow{2}{*}{ Lithofacies } & & \multirow{2}{*}{ 亨 } & \multirow{2}{*}{\multicolumn{2}{|c|}{ 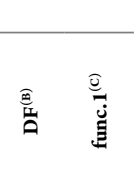 }} & \multirow[b]{2}{*}{ 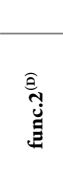 } \\
\hline & & $\overbrace{\frac{1}{4}}^{m}$ & $\widehat{\widehat{\sigma}}$ & $\widehat{\widehat{o}}$ & & $\frac{\xi}{\stackrel{5}{0}}$ & $\frac{\ddot{g}}{0}$ & $\frac{\ddot{g}}{0}$ & $\frac{0^{m}}{q^{n}}$ & $\begin{array}{l}0 \\
\tilde{Z}^{-1}\end{array}$ & ల & ฉి & $\begin{array}{l}0 \\
\tilde{Z}^{-1}\end{array}$ & 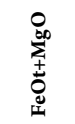 & 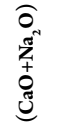 & $\overbrace{\substack{00 \\
\sum}}$ & $\rho^{N}$ & & & & \\
\hline SM51 & & 0.36 & -0.72 & 0.07 & 63.46 & 0.2 & 0.04 & 0.07 & 2.3 & 5.31 & 0.67 & 2.35 & 8.20 & 12.37 & 3.29 & 11.37 & 0.43 & 7.43 & 0.50 & 0.53 & -51.25 \\
\hline $\mathrm{T} 171 \mathrm{C}$ & & 0.60 & -0.23 & 0.36 & 46.62 & 0.15 & 0.14 & 0.03 & 3.94 & 1.69 & 0.55 & 2.94 & 4.30 & 10.53 & 1.86 & 9.74 & 0.25 & 5.86 & -1.28 & 0.26 & -45.66 \\
\hline T3720 & & 0.70 & 0.18 & 0.35 & 52.66 & 0.13 & 0.09 & 0.02 & 5.05 & 0.66 & 0.84 & 3.26 & 4.80 & 8.86 & 1.77 & 7.94 & 0.2 & 2.09 & 2.72 & 0.34 & -38.76 \\
\hline SM20 & Carbonate & 0.78 & 0.09 & 0.25 & 48.68 & 0.11 & 0.09 & 0.02 & 6.01 & 0.82 & 0.37 & 3.34 & 5.10 & 6.58 & 1.44 & 5.85 & 0.17 & -0.43 & 1.07 & 1.29 & -44.06 \\
\hline TM1085 & schist & 0.72 & 0.05 & 0.23 & 52.36 & 0.13 & 0.09 & 0.03 & 5.21 & 0.89 & 0.53 & 3.42 & 5.30 & 6.63 & 2.26 & 5.82 & 0.19 & -0.60 & 1.19 & 0.45 & -44.57 \\
\hline TM1160 & & 0.76 & 0.09 & 0.28 & 51.58 & 0.12 & 0.09 & 0.02 & 5.72 & 0.81 & 0.42 & 3.43 & 4.90 & 6.58 & 1.85 & 5.84 & 0.17 & -0.54 & 1.29 & 0.29 & -44.07 \\
\hline T185 & & 0.73 & -0.02 & 0.14 & 56.12 & 0.13 & 0.07 & 0.03 & 5.37 & 1.04 & 0.58 & 3.49 & 4.70 & 5.77 & 2.41 & 5.13 & 0.19 & 0.27 & 1.26 & 0.27 & -44.32 \\
\hline TM2418 & & 0.80 & -0.27 & 0.13 & 60.57 & 0.11 & 0.05 & 0.03 & 6.32 & 1.86 & 0.47 & 3.61 & 4.00 & 5.57 & 2.48 & 5.01 & 0.16 & 0.68 & 0.65 & -1.14 & -44.63 \\
\hline SP4A & & 0.74 & 0.07 & 0.25 & 52.05 & 0.12 & 0.08 & 0.03 & 5.54 & 0.86 & 0.90 & 3.53 & 6.96 & 7.48 & 2.55 & 6.82 & 0.18 & -1.71 & 1.68 & 0.71 & -46.88 \\
\hline SP4B & & 0.61 & -0.10 & 0.10 & 55.93 & 0.16 & 0.07 & 0.05 & 4.09 & 1.25 & 2.58 & 3.26 & 8.4 & 8.78 & 3.48 & 8.03 & 0.24 & -0.77 & 1.51 & 1.35 & -48.07 \\
\hline SP4C & & 0.57 & -0.22 & 0.11 & 55.69 & 0.16 & 0.08 & 0.05 & 3.71 & 1.64 & 1.80 & 3.12 & 8.33 & 10.21 & 3.60 & 9.42 & 0.27 & 6.06 & 0.27 & 0.95 & -48.56 \\
\hline SP4D & & 0.81 & 0.38 & 0.29 & 57.38 & 0.12 & 0.07 & 0.02 & 6.44 & 0.42 & 1.04 & 3.84 & 5.25 & 4.22 & 2.73 & 3.73 & 0.16 & -2.02 & 2.63 & -0.30 & -43.04 \\
\hline SP4E & & 0.68 & -0.06 & 0.03 & 57.21 & 0.14 & 0.07 & 0.04 & 4.78 & 1.14 & 2.67 & 3.5 & 7.49 & 6.39 & 3.59 & 5.8 & 0.21 & -1.05 & 1.58 & 0.53 & -46.56 \\
\hline SP4F & Salinas & 0.79 & 0.02 & 0.09 & 54.49 & 0.12 & 0.06 & 0.03 & 6.16 & 0.95 & 1.35 & 3.72 & 6.70 & 5.37 & 3.02 & 4.78 & 0.16 & -2.60 & 1.06 & -0.35 & -45.97 \\
\hline SP4G & Type-area & 0.69 & -0.05 & -0.03 & 54.86 & 0.14 & 0.07 & 0.04 & 4.86 & 1.12 & 1.33 & 3.52 & 7.73 & 5.56 & 3.18 & 5.04 & 0.21 & -0.76 & 1.49 & 0.61 & -47.59 \\
\hline SP4H & (metawacke) & 0.75 & 0.11 & 0.13 & 51.52 & 0.13 & 0.08 & 0.04 & 5.66 & 0.78 & 1.21 & 3.61 & 7.55 & 5.98 & 2.49 & 5.5 & 0.18 & -1.54 & 2.15 & 0.61 & -46.84 \\
\hline SP4I & & 0.58 & -0.35 & 0.05 & 59.36 & 0.16 & 0.05 & 0.06 & 3.82 & 2.24 & 2.37 & 3.19 & 7.8 & 9.28 & 4.89 & 8.48 & 0.26 & -0.01 & 0.86 & 0.48 & -49.10 \\
\hline SP4J & & 0.80 & 0.31 & 0.39 & 50.91 & 0.12 & 0.09 & 0.02 & 6.29 & 0.49 & 0.42 & 3.69 & 5.48 & 5.76 & 2.11 & 5.26 & 0.16 & -1.71 & 2.49 & 0.22 & -45.50 \\
\hline SP5A & & 0.63 & 0.10 & 0.04 & 50.8 & 0.15 & 0.11 & 0.04 & 4.22 & 0.79 & 0.82 & 3.27 & 8.18 & 6.22 & 2.34 & 5.66 & 0.24 & -0.36 & 2.01 & 1.69 & -46.51 \\
\hline SP5B & & 0.64 & -0.41 & 0.03 & 53.52 & 0.14 & 0.06 & 0.06 & 4.4 & 2.57 & 1.51 & 3.25 & 8.20 & 9.32 & 3.82 & 8.66 & 0.23 & 0.13 & 0.63 & 0.61 & -50.42 \\
\hline SP5C & & 0.71 & 0.59 & 0.35 & 52.07 & 0.14 & 0.11 & 0.02 & 5.13 & 0.26 & 0.83 & 3.62 & 7.15 & 4.68 & 2.05 & 4.29 & 0.20 & -2.34 & 3.29 & - & - \\
\hline SP5D & & 0.63 & 0.10 & 0.08 & 50.65 & 0.15 & 0.11 & 0.04 & 4.22 & 0.79 & 0.81 & 3.31 & 8.34 & 6.61 & 2.33 & 6.05 & 0.24 & -0.99 & 1.77 & - & - \\
\hline GSM1T8* & & 0.49 & -0.60 & 0.13 & 66.79 & 0.19 & 0.04 & 0.06 & 3.12 & 4.00 & 3.00 & 2.97 & 6.50 & 10.7 & 8.26 & 9.96 & 0.32 & 6.77 & -1.28 & -2.51 & -46.03 \\
\hline GSM4T8* & & 0.58 & -0.30 & 0.24 & 67.91 & 0.16 & 0.04 & 0.04 & 3.84 & 2.00 & 6.51 & 3.19 & 5.70 & 10.35 & 7.12 & 9.43 & 0.26 & 0.19 & 1.58 & -1.56 & -43.68 \\
\hline GSM5T8* & & 0.59 & -0.27 & 0.23 & 67.9 & 0.16 & 0.04 & 0.04 & 3.9 & 1.85 & 6.50 & 3.24 & 5.70 & 9.84 & 6.92 & 8.91 & 0.26 & 0.36 & 1.83 & -1.49 & -44.03 \\
\hline GSM6T8* & Muscovite & 0.6 & -0.27 & 0.25 & 69.22 & 0.16 & 0.03 & 0.04 & 3.96 & 1.84 & 8.97 & 3.25 & 5.40 & 9.65 & 7.49 & 8.85 & 0.25 & 0.10 & 1.93 & -1.73 & -43.63 \\
\hline $\mathrm{T} 171 \mathrm{~B}$ & schist & 0.49 & -0.34 & 0.18 & 60.85 & 0.18 & 0.06 & 0.05 & 3.08 & 2.17 & 2.38 & 2.87 & 7.30 & 12.33 & 4.77 & 11.44 & 0.32 & 6.37 & 0.38 & -0.62 & -46.52 \\
\hline T168 & & 0.49 & -0.56 & 0.13 & 54.99 & 0.18 & 0.05 & 0.05 & 3.09 & 3.64 & 2.11 & 2.91 & 6.50 & 11.42 & 5.88 & 10.61 & 0.32 & 6.68 & -1.43 & -1.57 & -46.73 \\
\hline TM1091 & & 0.53 & -0.15 & 0.23 & 56.86 & 0.17 & 0.09 & 0.04 & 3.39 & 1.41 & 1.43 & 2.95 & 7.00 & 11.17 & 3.35 & 10.28 & 0.3 & 4.86 & -1.67 & -1.30 & -43.38 \\
\hline GSM3T11* & Quartzose & 0.9 & 0.39 & 0.40 & 52.57 & 0.1 & 0.07 & 0.01 & 7.94 & 0.41 & 1.00 & 3.89 & 4.50 & 5.19 & 2.18 & 4.54 & 0.13 & -1.23 & 2.34 & 0.27 & -42.13 \\
\hline GSM4T11* & metawacke & 0.9 & 0.39 & 0.34 & 51.22 & 0.1 & 0.08 & 0.02 & 7.94 & 0.41 & 1.10 & 3.89 & 5.20 & 5 & 2.09 & 4.38 & 0.13 & -2.09 & 2.10 & 0.49 & -42.33 \\
\hline GSM2T10* & & 0.72 & 0.08 & 0.34 & 57 & 0.13 & 0.07 & 0.03 & 5.21 & 0.83 & 0.69 & 3.52 & 5.30 & 7.17 & 3.00 & 6.41 & 0.19 & -1.82 & 1.47 & -0.48 & -44.64 \\
\hline GSM4T5* & & 0.59 & -0.27 & 0.22 & 64.45 & 0.16 & 0.05 & 0.04 & 3.86 & 1.86 & 4.44 & 3.17 & 6.30 & 10.69 & 5.80 & 9.59 & 0.26 & 0.25 & 1.41 & -0.56 & -46.37 \\
\hline GSM5T5* & & 0.61 & -0.18 & 0.28 & 66.81 & 0.16 & 0.04 & 0.04 & 4.04 & 1.50 & 5.09 & 3.26 & 5.50 & 9.85 & 5.90 & 8.96 & 0.25 & 0.07 & 1.86 & -0.08 & -45.83 \\
\hline $\mathrm{R} 13$ & & 0.51 & -0.36 & 0.17 & 61.29 & 0.18 & 0.06 & 0.05 & 3.21 & 2.30 & 1.33 & 2.94 & 6.60 & 10.74 & 4.16 & 9.96 & 0.31 & 5.91 & -1.35 & -0.53 & -48.07 \\
\hline TM1084 & & 0.53 & -0.20 & 0.12 & 56.94 & 0.17 & 0.08 & 0.05 & 3.39 & 1.57 & 1.13 & 3 & 7.20 & 9. & 3.47 & 8.37 & 0.3 & 5.27 & -1.15 & 0.09 & -48.01 \\
\hline T3721 & Quartz- & 0.57 & 0.10 & 0.40 & 54.06 & 0.16 & 0.11 & 0.03 & 3. & 0.79 & 1.00 & 3.08 & 6.10 & 11.07 & 2.41 & 10.28 & 0.27 & 4.33 & -0.94 & 1.37 & -43.71 \\
\hline T192A & biotite & 0.54 & -0.25 & 0.25 & 67.1 & 0.17 & 0.04 & 0.04 & 3.48 & 1.77 & 5.09 & 3.1 & 6.10 & 10.72 & 6.43 & 9.8 & 0.29 & 0.31 & 1.82 & -0.36 & -46.39 \\
\hline T210 & schist & 0.56 & -0.21 & 0.23 & 62.42 & 0.17 & 0.06 & 0.04 & 3.67 & 1.61 & 2.57 & 3.1 & 6.00 & 10.71 & 4.57 & 9.85 & 0.27 & 1.04 & 1.79 & -0.27 & -45.23 \\
\hline $\mathrm{T} 170 \mathrm{C}$ & & 0.56 & -0.42 & 0.15 & 60.55 & 0.17 & 0.06 & 0.05 & 3.67 & 2.61 & 1.12 & 3.1 & 6.50 & 10.43 & 3.84 & 9.57 & 0.27 & 7.37 & 1.55 & -0.05 & -48.32 \\
\hline TM1097 & & 0.63 & -0.11 & 0.21 & 59.01 & 0.15 & 0.06 & 0.04 & 4.25 & 1.29 & 2.17 & 3.21 & 6.40 & 9.45 & 3.78 & 8.45 & 0.24 & 0.02 & 1.50 & 0.43 & -45.86 \\
\hline T3706 & & 0.61 & -0.17 & 0.18 & 65.97 & 0.15 & 0.04 & 0.04 & 4.06 & 1.48 & 9.36 & 3.21 & 5.70 & 10.49 & 5.70 & 9.51 & 0.25 & 1.23 & 2.02 & 0.53 & -43.64 \\
\hline R8B & & 0.61 & -0.23 & 0.16 & 57.24 & 0.16 & 0.07 & 0.04 & 4.03 & 1.71 & 1.19 & 3.25 & 6.50 & 9.17 & 3.58 & 8.44 & 0.25 & -0.03 & 1.07 & 0.22 & -46.77 \\
\hline T3722 & & 0.64 & -0.23 & 0.25 & 66.54 & 0.15 & 0.04 & 0.03 & 4.33 & 1.68 & 4.65 & 3.32 & 5.10 & 9.88 & 5.86 & 8.97 & 0.23 & 0.76 & 1.63 & 0.20 & -45.10 \\
\hline T3716 & & 0.67 & -0.52 & 0.15 & 67.27 & 0.14 & 0.03 & 0.04 & 4.7 & 3.33 & 11.22 & 3.34 & 5.20 & 11.00 & 8.82 & 10.27 & 0.21 & 1.98 & 1.40 & -0.50 & -44.92 \\
\hline
\end{tabular}

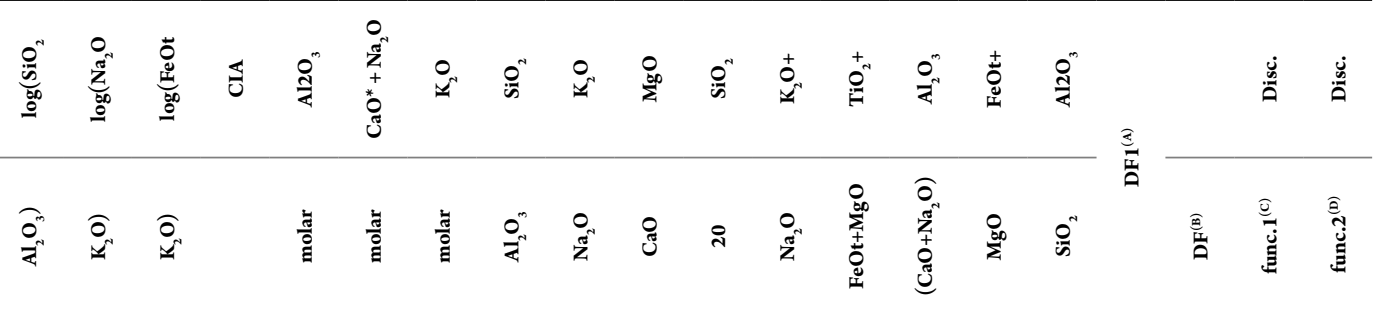

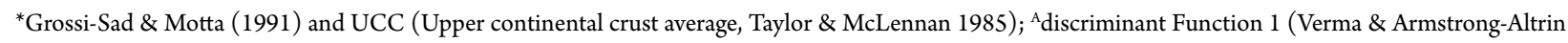
2013); ${ }^{B}$ discriminant Function 2 (Verma \& Armstrong-Altrin 2013); ${ }^{C}$ discriminant Function 1 (Bhatia 1985); ${ }^{D}$ discriminant Function 2 (Bhatia 1985). 
a well-defined trend with relatively low CIA values close to the plagioclase corner and increase of $\mathrm{Al}_{2} \mathrm{O}_{3}, \mathrm{~K}_{2} \mathrm{O}$ and CIA values (Fig. 5). The lower segment of this trend, with CIA from 46 to 60 , includes the metawackes of the type area and most of their deformed equivalents (the carbonate schist and quartzose metawacke). The upper segment of the trend (CIA from 60 to 70) mainly includes the quartz-biotite and muscovite schists, reflecting the more pelitic $\left(\mathrm{Al}_{2} \mathrm{O}_{3}\right.$ - and $\mathrm{K}$-rich $)$ fractions present in these rocks. This trend suggests sediment sources originally rich in intermediate to felsic igneous rocks. The upper part of the trend may reflect clay-rich fractions directly eroded and transported from more weathered sources and/or the conversion of feldspars into clay during diagenetic processes. In turn, metawackes from the type area, carbonate schist and quartzose

Table 3. Trace element (in ppm) compositions of clastic metasedimentary rocks from the Salinas Formation.

\begin{tabular}{|c|c|c|c|c|c|c|c|c|c|c|c|c|c|c|c|c|c|c|c|c|c|}
\hline Sample & Lithofacies & $\mathbf{L a}$ & $\mathrm{Ce}$ & Nd & Sm & Eu & Gd & Dy & Ho & Er & $\mathbf{Y b}$ & $\mathbf{L u}$ & $\mathbf{U}$ & Th & $\mathrm{Zr}$ & Sc & Co & $\begin{array}{l}\mathbf{E u} / \\
\mathbf{E u}^{*}\end{array}$ & $\mathrm{Zr} / 10$ & $\mathbf{T i} / \mathbf{Z r}$ & $\mathbf{L a} / \mathbf{S c}$ \\
\hline SM51 & & 44.11 & 97.23 & 42.94 & 8.37 & 1.53 & 6.01 & 4.91 & 1 & 2.33 & 2.02 & 0.28 & - & 13 & 290 & 22 & - & 0.05 & 29 & 16.5 & 2.01 \\
\hline $\mathrm{T} 171 \mathrm{C}$ & & 27.15 & 61.52 & 28.79 & 5.98 & 1.1 & 4.71 & 5.26 & 1.19 & 3.18 & 2.95 & 0.4 & - & - & - & - & - & 0.07 & - & - & - \\
\hline T3720 & & 50.68 & 110.5 & 54.08 & 10.63 & 1.91 & 8.46 & 8.89 & 1.87 & 5.35 & 4.84 & 0.63 & - & 13 & 370 & 19 & - & 0.04 & 37 & 14.06 & 2.67 \\
\hline SM20 & Carbonate & 34.97 & 76.69 & 36.42 & 7.36 & 1.18 & 5.55 & 5.6 & 1.25 & 3.26 & 3.19 & 0.43 & - & 15 & 390 & 15 & - & 0.05 & 39 & 16.81 & 2.33 \\
\hline TM1085 & schist & 43.1 & 83.1 & 37.6 & 7.1 & 1.4 & 6.3 & 4.4 & 0.93 & 2.3 & 1.9 & 0.25 & - & - & 430 & - & - & 0.05 & 43 & 13.74 & - \\
\hline TM1160 & & 30.3 & 50.4 & 27 & 5.3 & 0.98 & 5.1 & 3.9 & 0.83 & 2.1 & 1.9 & 0.25 & - & - & 400 & - & - & 0.07 & 40 & 16.17 & - \\
\hline T185 & & 30.8 & 65.93 & 30.85 & 6.09 & 1.2 & 4.51 & 4.54 & 0.99 & 2.67 & 2.54 & 0.33 & - & 14 & 280 & 17 & - & 0.06 & 28 & 26.71 & 1.81 \\
\hline TM2418 & & 43.1 & 82 & 37.4 & 7.2 & 1.3 & 6.3 & 4.1 & 0.86 & 2.1 & 1.9 & 0.26 & - & - & 460 & - & - & 0.05 & 46 & 18.58 & - \\
\hline SP4A & & 84.5 & 161.53 & 60.53 & 10.33 & 16.92 & 7.95 & 6.59 & 1.27 & 3.76 & 3.64 & 0.54 & 4.45 & 27.29 & 522.3 & 9 & 35.16 & 0.04 & 52 & 13.95 & 9.39 \\
\hline SP4B & & 41.43 & 88.04 & 36.66 & 74.23 & 1.32 & 63.1 & 5.99 & 1.2 & 3.6 & 3.23 & 0.49 & 3.45 & 19.86 & 192.51 & 13 & 24.35 & 0.05 & 19 & 32.97 & 3.19 \\
\hline SP4C & & 60.55 & 105.99 & 47.14 & 8.43 & 1.54 & 6.77 & 5.61 & 1.08 & 3.23 & 3.01 & 0.44 & 2.18 & 20.77 & 180.48 & 15 & 23.89 & 0.05 & 18 & 33.4 & 4.04 \\
\hline SP4D & & 43.95 & 80.63 & 32.74 & 5.59 & 1.11 & 4.24 & 3.75 & 0.71 & 2.07 & 2.03 & 0.31 & 3.27 & 15.83 & 267.23 & 7 & 28.60 & 0.07 & 27 & 36.55 & 6.28 \\
\hline SP4E & & 50 & 87.13 & 38.82 & 6.95 & 1.36 & 5.92 & 5.2 & 0.99 & 2.93 & 2.77 & 0.39 & 2.25 & 16.96 & 249.31 & 9 & 19.38 & 0.06 & 25 & 32.48 & 5.56 \\
\hline SP4F & Salinas & 71.9 & 136.02 & 50.79 & 8.4 & 1.43 & 6.68 & 5.66 & 1.1 & 3.1 & 2.91 & 0.44 & 3.00 & 23.49 & 393.36 & 8 & 44.81 & 0.05 & 39 & 20.73 & 8.99 \\
\hline SP4G & Type-area & 49.02 & 95.62 & 37.38 & 6.52 & 1.28 & 5.06 & 4.75 & 0.9 & 2.77 & 2.55 & 0.38 & 2.46 & 14.63 & 223.26 & 8 & 25.14 & 0.06 & 22 & 40.99 & 6.13 \\
\hline SP4H & (metawacke) & 38.94 & 77.25 & 30.45 & 5.54 & 1.13 & 4.47 & 4.0 & 0.79 & 2.3 & 2.13 & 0.31 & 2.10 & 13.20 & 189.2 & 8 & 18.38 & 0.07 & 19 & 52.92 & 4.87 \\
\hline SP4I & & 44.3 & 92.37 & 38.68 & 7.39 & 1.41 & 6.21 & 6.18 & 1.21 & 3.64 & 3.3 & 0.51 & 3.44 & 21.12 & 179.05 & 15 & 25.49 & 0.05 & 18 & 33.37 & 2.95 \\
\hline SP4J & & 56.56 & 107.6 & 39.91 & 6.79 & 1.2 & 5.21 & 4.49 & 0.87 & 2.61 & 2.53 & 0.38 & 3.18 & 18.78 & 320.99 & 7 & 35.94 & 0.06 & 32 & 29.88 & 8.08 \\
\hline SP5A & & 41.73 & 84.27 & 36.77 & 7.08 & 1.37 & 5.78 & 5.31 & 1.06 & 3.15 & 2.91 & 0.44 & 2.20 & 18.65 & 242.77 & 11 & 35.82 & 0.06 & 24 & 34.95 & 3.79 \\
\hline SP5B & & 37.82 & 77.98 & 32.94 & 6.66 & 1.41 & 5.56 & 5.03 & 1.02 & 3.09 & 2.9 & 0.44 & 3.13 & 15.6 & 203.59 & 13 & 26.11 & 0.06 & 20 & 35.4 & 2.91 \\
\hline SP5C & & 20.27 & 27.43 & 17.94 & 3.9 & 0.67 & 3.44 & 4.18 & 0.91 & 3.14 & 3.29 & 0.53 & 4.72 & 17.04 & 200.32 & 8 & 21.26 & 0.1 & 20 & 61.11 & 2.53 \\
\hline SP5D & & 42.74 & 86.8 & 37.57 & 7.22 & 1.4 & 6.06 & 5.51 & 1.08 & 3.2 & 2.96 & 0.44 & 2.25 & 18.99 & 243.47 & 11 & 44.10 & 0.05 & 24 & 34.98 & 3.89 \\
\hline GSM1T8* & & 42.61 & 93.21 & 40.4 & 7.68 & 1.41 & 6.17 & 6.2 & 1.25 & 3.4 & 3.21 & 0.39 & - & - & - & - & 53 & 0.05 & - & - & - \\
\hline GSM4T8* & & 11.63 & 24.05 & 9.795 & 1.97 & 0.50 & 2.14 & 3.13 & 0.69 & 2.17 & 2.63 & 0.37 & - & - & - & - & 13 & 0.20 & - & - & - \\
\hline GSM5T8* & & 38.36 & 83.94 & 35.89 & 6.71 & 1.23 & 5.1 & 5.14 & 1.05 & 2.96 & 3.26 & 0.41 & - & - & - & - & 13 & 0.06 & - & - & - \\
\hline GSM6T8* & Muscovite & 32.43 & 71.29 & 31.29 & 5.94 & 1.13 & 4.62 & 4.8 & 1 & 2.89 & 2.96 & 0.37 & - & - & - & - & 20 & 0.07 & - & - & - \\
\hline $\mathrm{T} 171 \mathrm{~B}$ & schist & 56.93 & 86.46 & 55.55 & 10.27 & 1.77 & 7.06 & 5.34 & 1.08 & 2.47 & 1.8 & 0.23 & - & 7 & 200 & 23 & - & 0.04 & & 32.78 & 2.48 \\
\hline T168 & & 26.81 & 61.37 & 26.66 & 5.4 & 0.99 & 3.85 & 3.75 & 0.83 & 2.26 & 2.42 & 0.34 & - & 12 & 200 & 22 & - & 0.07 & 20 & 29.54 & 1.22 \\
\hline TM1091 & & 22.1 & 59.1 & 21.7 & 4.5 & 0.88 & 4.3 & 3.8 & 0.84 & 2.4 & 2.6 & 0.35 & - & - & 310 & - & - & 0.09 & 31 & 17.35 & \\
\hline GSM3T11* & Quartzose & 24.99 & 60.18 & 28.61 & 5.28 & 1.01 & 3.73 & 3.84 & 0.79 & 2.2 & 2.15 & 0.26 & - & - & - & - & 7 & 0.07 & - & - & - \\
\hline GSM4T11* & metawacke & 22.07 & 45.34 & 20.41 & 5.15 & 0.9 & 4.58 & 4.1 & 0.82 & 2.17 & 2.52 & 0.36 & - & - & - & - & - & 0.08 & - & - & - \\
\hline GSM2T10* & & 35.69 & 79.9 & 34.69 & 6.46 & 1.09 & 4.72 & 4.16 & 0.84 & 2.29 & 2.53 & 0.32 & - & - & - & - & 9 & 0.06 & - & - & - \\
\hline GSM4T5* & & 42.14 & 92.28 & 40.43 & 8.03 & 1.41 & 6.29 & 6.74 & 1.39 & 3.97 & 4.04 & 0.54 & - & - & - & - & 18 & 0.05 & - & - & - \\
\hline GSM5T5* & & 29.65 & 68.12 & 33.03 & 6.24 & 1.19 & 4.6 & 4.89 & 0.99 & 2.72 & 2.66 & 0.34 & - & - & - & - & 36 & 0.06 & - & - & - \\
\hline R13 & & 29.03 & 68.5 & 32.01 & 6.37 & 1.22 & 4.6 & 4.29 & 0.88 & 2.31 & 2.32 & 0.34 & - & 13 & 200 & 18 & - & 0.06 & 20 & 31.49 & 1.61 \\
\hline TM1084 & & 43.6 & 92.3 & 40.6 & 8 & 1.5 & 6.5 & 4.2 & 0.84 & 2.1 & 2.2 & 0.3 & - & - & 500 & - & - & 0.05 & 50 & 8.7 & \\
\hline T3721 & Quartz- & 32.69 & 78.9 & 34.35 & 6.57 & 1.22 & 4.9 & 5.16 & 1.06 & 3.06 & 3.13 & 0.42 & - & - & - & - & - & 0.06 & - & - & - \\
\hline T192A & biotite & 30.67 & 67.25 & 31.08 & 6.37 & 1.25 & 4.8 & 4.75 & 0.98 & 2.67 & 2.81 & 0.39 & - & 11 & 230 & 19 & - & 0.06 & 23 & 26.68 & 1.61 \\
\hline $\mathrm{T} 210$ & schist & 35.8 & 71.05 & 33.21 & 6.88 & 1.24 & 5.16 & 5.19 & 1.21 & 2.98 & 3.13 & 0.43 & - & 18 & 210 & 18 & - & 0.06 & 21 & 27.46 & 1.99 \\
\hline $\mathrm{T} 170 \mathrm{C}$ & & 44.96 & 89.69 & 43.06 & 8.33 & 1.56 & 6.17 & 6.54 & 1.4 & 3.51 & 3.01 & 0.37 & - & - & - & - & - & 0.05 & - & - & - \\
\hline T3706 & & 25.13 & 52.38 & 24.62 & 4.91 & 1.05 & 3.66 & 4.29 & 0.90 & 2.72 & 2.89 & 0.39 & - & 13 & 240 & 22 & - & 0.08 & 24 & 21.68 & 1.14 \\
\hline R8B & & 34.99 & 71.9 & 33.13 & 6.23 & 1.18 & 4.46 & 4.08 & 0.84 & 2.16 & 1.88 & 0.23 & - & 13 & 220 & 17 & - & 0.06 & 22 & 25.3 & 2.06 \\
\hline T3722 & & 40.65 & 76.31 & 38.36 & 7.71 & 1.48 & 5.58 & 5.017 & 0.98 & 2.74 & 2.61 & 0.35 & - & 8 & 230 & 21 & - & 0.05 & 23 & 24.2 & 1.94 \\
\hline T3716 & & 29.11 & 63.85 & 28.05 & 5.1 & 0.97 & 3.52 & 2.96 & 0.54 & 1.65 & 1.89 & 0.28 & - & 7 & 170 & 20 & - & 0.08 & 17 & 28.15 & 1.46 \\
\hline UCC & & 30 & 64 & 26 & 4.5 & 0.88 & 3.8 & 3.5 & 0.8 & 2.3 & 2.2 & 0.32 & 2.8 & 10.7 & 190 & 13.6 & 17 & 0.09 & 19 & 0.09 & 2.21 \\
\hline
\end{tabular}

${ }^{*}$ Grossi-Sad \& Motta (1991) and UCC (Upper continental crust average, Taylor \& McLennan 1985). 
metawacke retain detrital compositions relatively poor in clay fraction, as suggested by the presence of lithic fragments, as well as abundant quartz and plagioclase, instead of micas and other Al-rich silicates.

Salinas rocks have an average $\mathrm{SiO}_{2} / \mathrm{Al}_{2} \mathrm{O}_{3}$ ratio of 5 , and values between 2 and 8 , reflecting a high degree of immaturity, which is typical of deposition close to the source (cf. Zhang 2004), although it can also be associated with quick transport and/or cold climate conditions.

The sediment maturity index $\left(\mathrm{SiO}_{2} / \mathrm{Al}_{2} \mathrm{O}_{3}\right)$ can be compared with the relative contributions of K-rich components versus plagioclase $\left(\mathrm{K}_{2} \mathrm{O} / \mathrm{Na}_{2} \mathrm{O}\right)$ and iron-magnesium minerals versus plagioclase $(\mathrm{MgO} / \mathrm{CaO})$, using the triangular diagram proposed by Pedrosa-Soares (1995) (Fig. 6). Besides the correlation of metawackes from the type area with carbonate schist and quartzose metawacke (mostly of them plotting closer the $\mathrm{SiO}_{2} / \mathrm{Al}_{2} \mathrm{O}_{3}$ vertex in relation to quartz-biotite schist and muscovite schist), the sample set follows a roughly curved trend from the more mature rocks toward the $\mathrm{MgO} / \mathrm{CaO}$ vertex. It suggests that detrital plagioclase and iron-magnesium minerals were important contributions even in the pelitic protoliths, and the K-rich contribution was mainly related to mica and/or clay fractions rather than detrital K-feldspar. The $\mathrm{CaO}$ component can be mainly ascribed to detrital plagioclase, even in the carbonate schist and metawackes, as they plot far from the $\mathrm{MgO} / \mathrm{CaO}$ vertex and tend to have a relatively narrow range of $\mathrm{K}_{2} \mathrm{O} / \mathrm{Na}_{2} \mathrm{O}$ ratios.

As expected from the field and petrographic studies, diagrams for lithological classification based on major element content (Pettijohn et al. 1972, Herron 1988) show that the studied rocks plot mostly in the graywacke and lithoarenite fields (Fig. 7). The relative high values of $\mathrm{SiO}_{2}$ (47-78\%), $\mathrm{Al}_{2} \mathrm{O}_{3}(10-20 \%)$ and $\mathrm{FeO}_{\mathrm{t}}(8-22 \%)$ reflect a terrigenous composition with significant argillaceous contribution. The higher

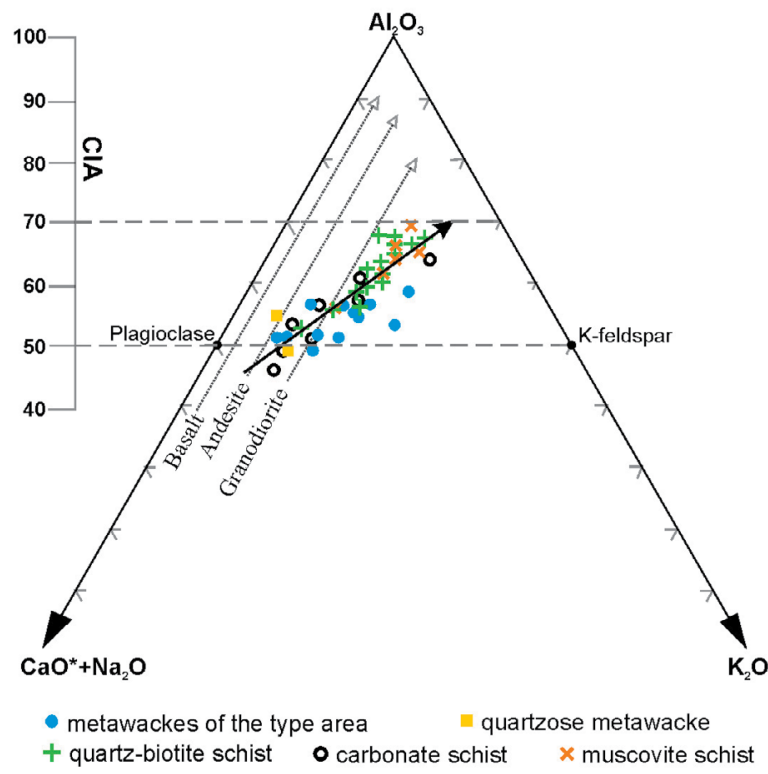

Figure 5. $\mathrm{Al}_{2} \mathrm{O}_{3}-\left(\mathrm{CaO}^{*}+\mathrm{Na}_{2} \mathrm{O}\right)-\mathrm{K}_{2} \mathrm{O}$ (in molar proportion) diagram for the Salinas samples, together with the chemical index of alteration (CIA) scale. The black arrow shows the compositional trend of the studied samples. The light grey dotted arrows indicate weathering trends of some common igneous rocks. content of silica corresponds to relatively quartz-rich and biotite-poor rocks, mostly the massive metawacke and quartzose metawacke, but also to some quartz-biotite schist and carbonate schist. The higher content of $\mathrm{Al}_{2} \mathrm{O}_{3}$ and $\mathrm{FeO}_{4}$ correlates with increasing modal values of biotite and other peraluminous Fe-rich silicates (e.g., garnet and staurolite), reflecting the importance of the pelitic (mostly clay) contribution. $\mathrm{TiO}_{2}$, $\mathrm{Al}_{2} \mathrm{O}_{3}, \mathrm{MgO}, \mathrm{P}_{2} \mathrm{O}_{5}, \mathrm{~K}_{2} \mathrm{O}, \mathrm{FeO}_{\mathrm{t}}$ and $\mathrm{MnO}$ are negatively correlated with $\mathrm{SiO}_{2}$, reflecting the decrease of silica with increase of the matrix proportion together with increase of the maturity index (Fig. 8). In contrast, $\mathrm{Na}_{2} \mathrm{O}$ exhibits a positive correlation with $\mathrm{SiO}_{2}$ (Fig. 4), and the higher $\mathrm{Na}_{2} \mathrm{O}$ values correspond to samples of metawacke from the Salinas type area and quartzose metawacke found in the Minas Novas corridor, which are generally the lithotypes richer in plagioclase. As the plagioclase found in those lithotypes is generally andesine to oligoclase, the lithochemical signature also points to sources rich in intermediate to felsic granitic rocks.

The large compositional spread of major elements as to the upper continental crust average suggests great variability in sediment maturity, involving a wide spectrum of mixtures from distinct contributions, possibly also reflecting distinct sources ( $c f$. Taylor \& McLennan 1985).

\section{Provenance and tectonic environment}

The low metamorphic grade of Salinas metawackes, in the type area and elsewhere (Figs. 1 and 2), suggests that the metamorphism has not strongly affected their primary chemical signatures, allowing the characterization of sedimentary protolith compositions and related tectonic environments. Furthermore, the high chemical immaturity (i.e., low $\mathrm{SiO}_{2} /$ $\mathrm{Al}_{2} \mathrm{O}_{3}$ ) and limited weathering of the source rocks (i.e., low CIA) allow us to assess the provenance of these rocks using petrographic and lithochemical features. The main sources for the Salinas sediments can be inferred from the relative abundance of their grains in thin section, in which the high

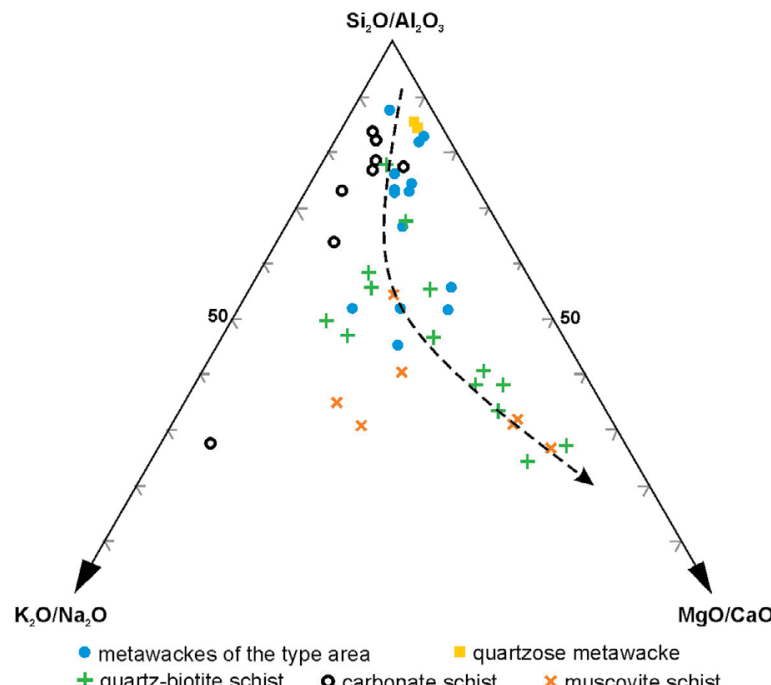

Figure 6. Chemical compositions of rocks of the Salinas Formation plotted in the $\mathrm{SiO}_{2} / \mathrm{Al}_{2} \mathrm{O}_{3}-\mathrm{K}_{2} \mathrm{O} / \mathrm{Na}_{2} \mathrm{O}-\mathrm{MgO} / \mathrm{CaO}$ diagram of Pedrosa-Soares (1995). The dashed black arrow follows the curved trend shown by the sample set. 
proportion of quartz and plagioclase in the metawackes may indicate tonalitic-granodioritic sources.

However, chemical modifications may occur due to the high mobility of some major elements, even at low-grade metamorphic conditions. Therefore, trace elements such as $\mathrm{La}, \mathrm{Sc}, \mathrm{Th}, \mathrm{Ti}, \mathrm{Zr}$ and $\mathrm{Co}$ are considered reliable indicators of tectonic environments (Bhatia 1985, Bhatia \& Crook 1986), and when combined with major elements, they can be used to better identify them. The rare earth element (REE) distribution patterns (Fig. 9) show some overall enrichment in the REE content, specially of the light REE (LREE), and intermediate to weakly negative Eu anomalies $\left(\mathrm{Eu} / \mathrm{Eu}^{*}=0.04-0.20\right)$, with the average upper to continental crust (UCC) curve roughly at the midpoint within the compositional range for Salinas Formation. The range of negative $\mathrm{Eu}$ anomalies $\left(\mathrm{Eu} / \mathrm{Eu}^{*}=\right.$ $0.04-0.20)$ overlaps the UCC corresponding value $\left(\mathrm{Eu} / \mathrm{Eu}^{*}=\right.$
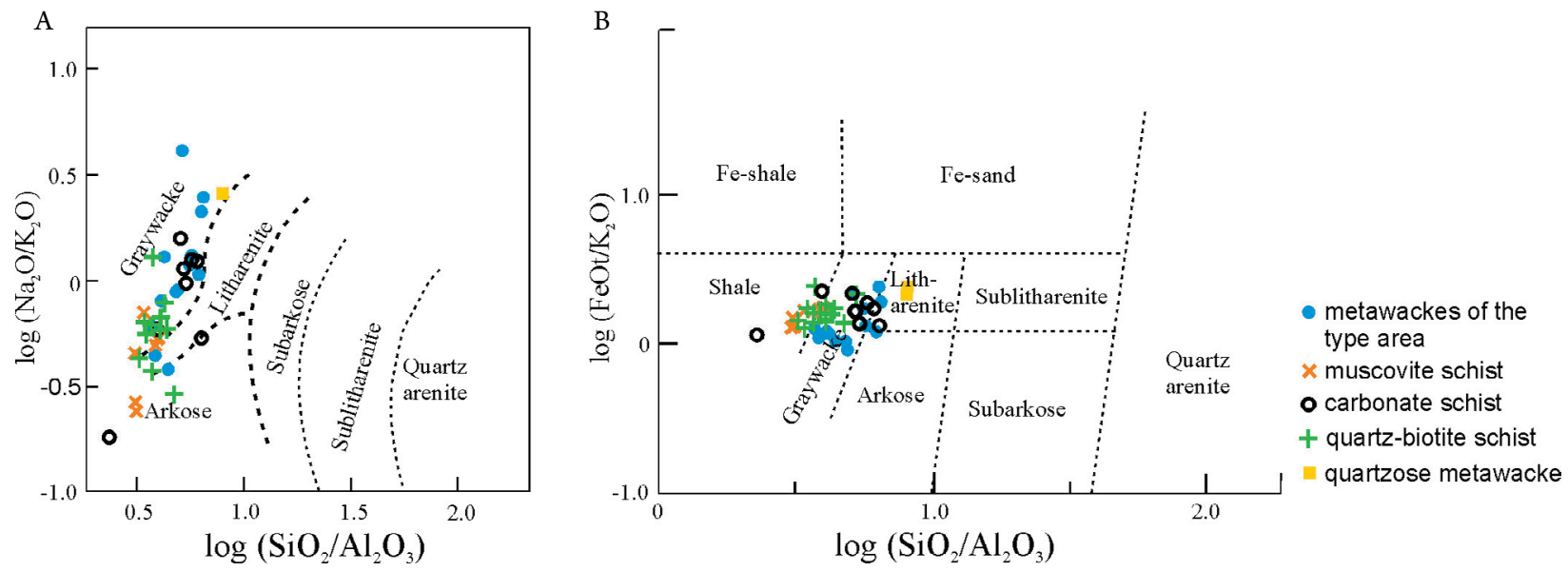

Figure 7. Chemical classification diagrams for rocks from the Salinas Formation: (a) $\mathrm{Log}\left(\mathrm{Na}_{2} \mathrm{O} / \mathrm{K}_{2} \mathrm{O}\right)$ versus $\log \left(\mathrm{SiO}_{2} / \mathrm{Al}_{2} \mathrm{O}_{3}\right)$ of $\mathrm{Pettijohn}$ et al. (1972); (b) $\mathrm{Log}(\mathrm{FeOt} / \mathrm{K} 2 \mathrm{O})$ versus $\log \left(\mathrm{SiO}_{2} / \mathrm{Al}_{2} \mathrm{O}_{3}\right)$ of Herron (1988).

A

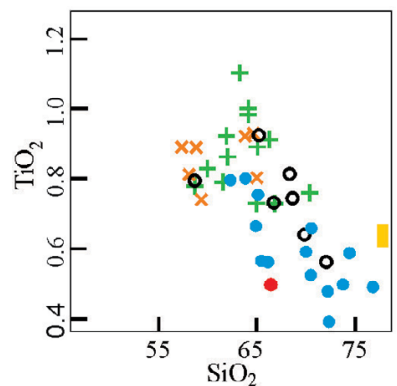

$\mathrm{D}$

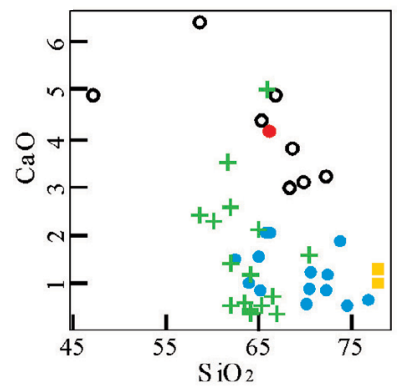

G

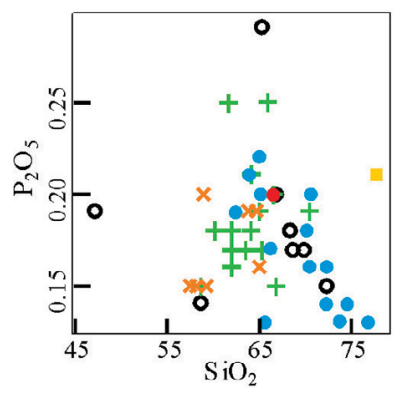

B

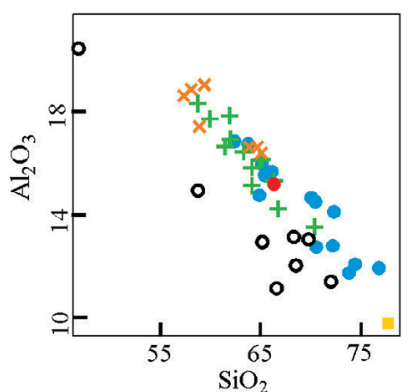

$\mathrm{E}$

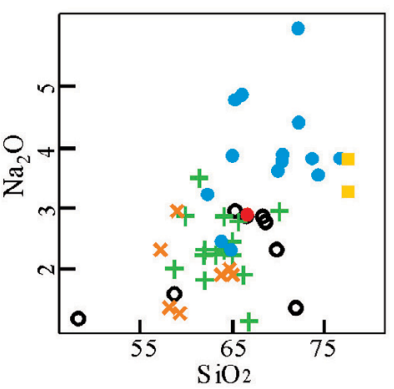

$\mathrm{H}$

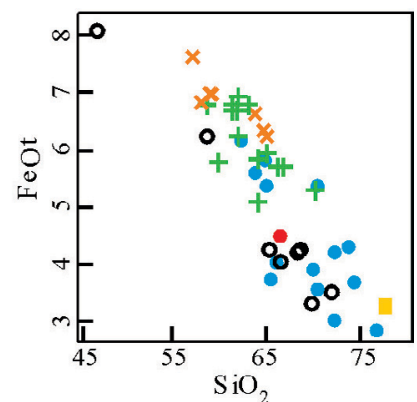

C

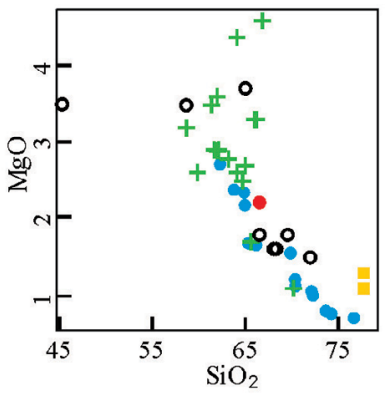

F
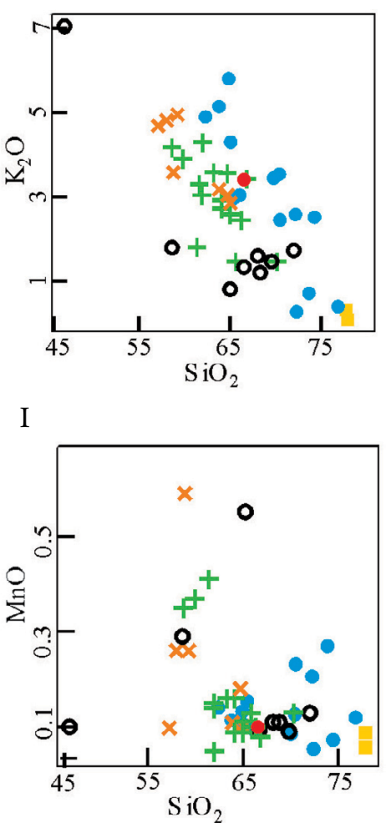

- metawackes of the type area

$x$ muscovite schist

- carbonate schist

+ quartz-biotite schist

- quartzose metawacke

- upper continental crust (UCC)

Figure 8. Bivariant diagrams of $\mathrm{SiO}_{2}$ versus (a) $\mathrm{TiO}_{2}$, (b) $\mathrm{Al}_{2} \mathrm{O}_{3}$, (c) $\mathrm{MgO}$, (d) $\mathrm{CaO},(\mathrm{e}) \mathrm{Na}_{2} \mathrm{O}$, (f) $\mathrm{K}_{2} \mathrm{O},(\mathrm{g}) \mathrm{P}_{2} \mathrm{O}_{5}$, (h) $\mathrm{FeO}_{\mathrm{t}}$; and (i) $\mathrm{MnO}$. Upper continental crust (UCC) values are from Taylor \& McLennan (1985). 


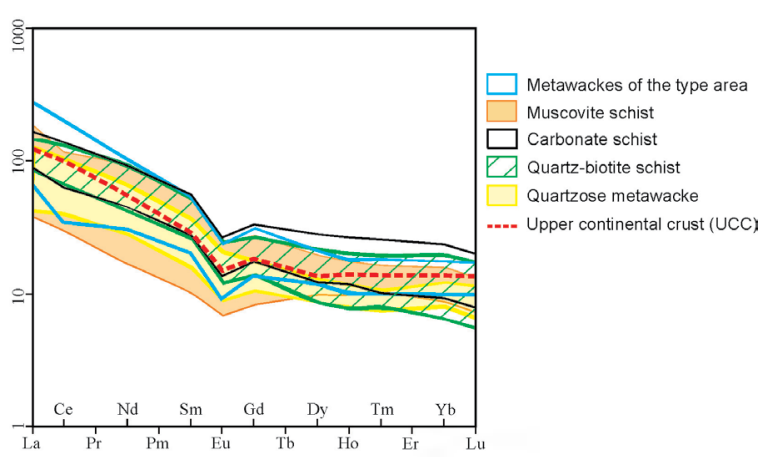

Figure 9. Chondrite-normalized REE patterns for the Salinas metawackes. Chondrite values from Boynton (1984), UCC values from Taylor \& McLennan (1985).

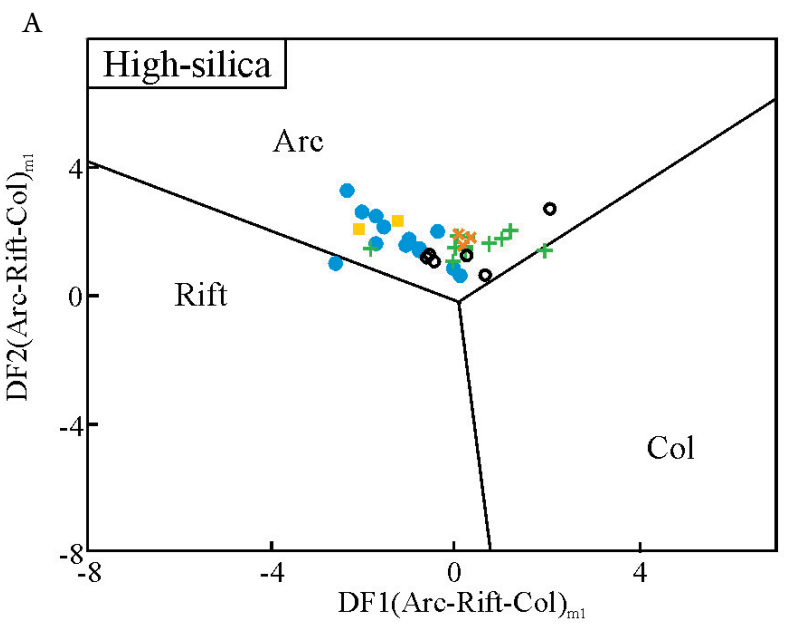

$0.09)$, suggesting contribution from predominantly felsic protoliths. Although these features are not conclusive, the general REE pattern tends to be close to those of mature active margins with continental magmatic arcs (Bhatia 1985, Taylor \& McLennan 1985, Sun et al. 2017). Despite some scattered distributions, discriminant diagrams for tectonic environments (Figs. 10 and 11) show that the Salinas rocks plot mainly in the fields of continental magmatic arc, active continental margin, collisional and oceanic island arc, but they never do it in the passive margin or rift fields.

Diagrams of Verma and Armstrong-Altrin (2013) have been used to infer tectonic setting of Precambrian clastic rocks. The high-silica diagram corresponds to a $\left(\mathrm{SiO}_{2}\right)_{\text {adj }}$ value

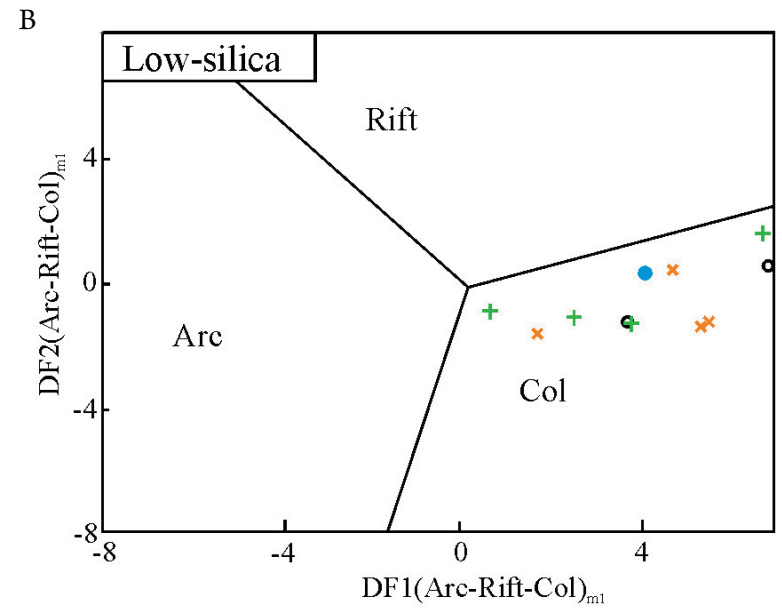

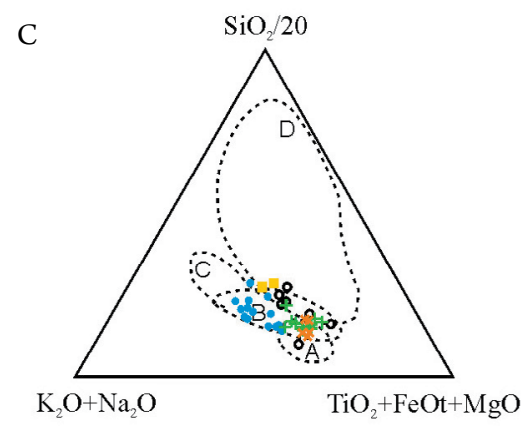

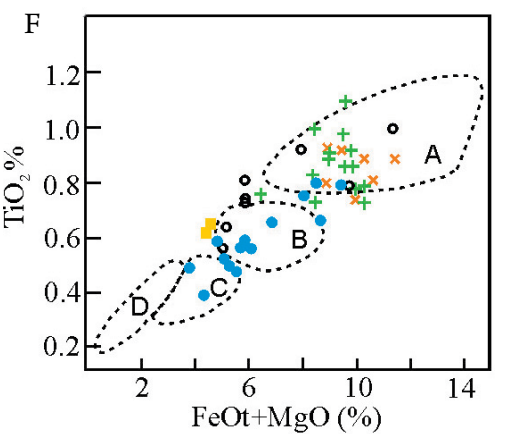

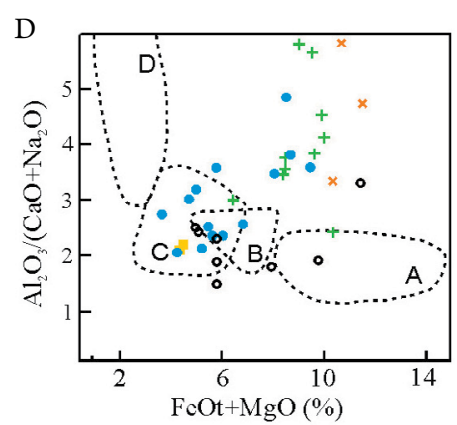

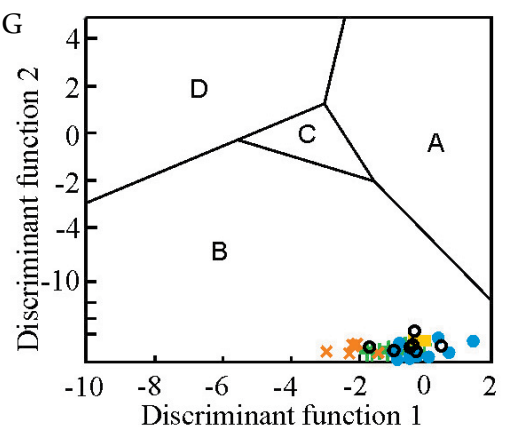

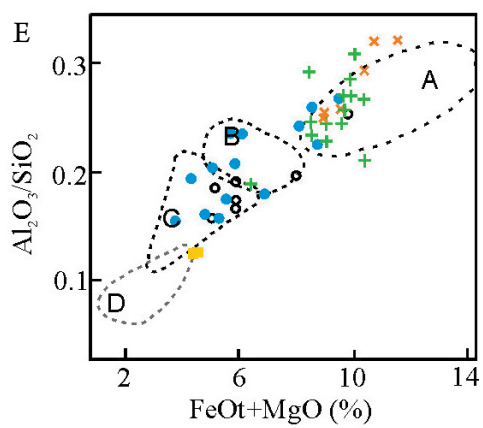

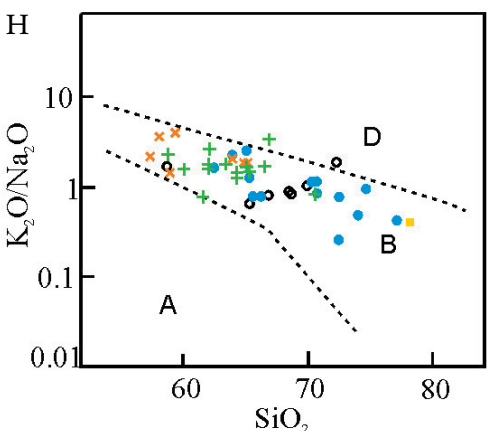

\footnotetext{
- metawackes of the type area

- quartzose metawacke

+ quartz-biotite schist 0 carbonate schist $\quad \times$ muscovite schist
}

Figure 10. Major-elements diagrams for provenance discrimination of protoliths of Salinas rocks: (a) Discriminant-function multidimensional diagram for high-silica sediments, DF1 versus DF2 of Verma \& Armstrong-Altrin (2013); (b) Discriminant-function multidimensional diagram for low-silica sediments, DF1 versus DF2 of Verma \& Armstrong-Altrin (2013); (c) Ternary diagram $\mathrm{K}_{2} \mathrm{O}+\mathrm{Na}_{2} \mathrm{O}-$ $\mathrm{SiO}_{2} / 20-\mathrm{TiO}_{2}+\mathrm{FeO}^{\mathrm{t}}+\mathrm{MgO}$ of Kroonenberg (1994); (d) $\mathrm{Al}_{2} \mathrm{O}_{3} /\left(\mathrm{CaO}+\mathrm{Na}_{2} \mathrm{O}\right)$ versus $\mathrm{FeO}^{\mathrm{t}}+\mathrm{MgO}$ of Bhatia (1985); (e) $\mathrm{Al}_{2} \mathrm{O}_{3} / \mathrm{SiO}_{2}$ versus $\mathrm{FeOt}+\mathrm{MgO}$ of Bhatia (1985); (f) $\mathrm{TiO}_{2}$ versus $\mathrm{FeOt}+\mathrm{MgO}$ of Bhatia (1985); (g) Discriminant function diagram from Bhatia (1985), Discriminant Function 2 versus Discriminant Function 1; (h) $\mathrm{K}_{2} \mathrm{O} / \mathrm{Na}_{2} \mathrm{O}$ of Roser \& Korsch (1986). Fields: Col- collisional; A, oceanic island arc; B, continental magmatic arc; $\mathrm{C}$, active continental margin; $\mathrm{D}$, passive continental margin. 
between 63 and 95\%, and low-silica diagram to a $\left(\mathrm{SiO}_{2}\right)_{\text {adj }}$ value between 35 and $63 \%$, in which $\left(\mathrm{SiO}_{2}\right)_{\text {adj }}$ refers to the $\mathrm{SiO}_{2}$ value obtained after volatile-free adjustment of the ten major-elements to $100 \mathrm{wt} \%$. Most studied samples belong to the high-silica type that plotted in the arc field (Fig. 10A). For the low-silica diagram, the remaining samples plotted in the collisional field (Fig. 10B). Comparing the composition of low-silica and high-silica types, the source rocks for the low-silica type are expressively more aluminous than for the high-silica samples. Thus, the percentage of sources rich in pelitic rocks should be higher for the low-silica samples. This signature could be derived from secondary sources as Macaúbas Group, Jequitinhonha Complex, and metavolcanic and meta-volcaniclastic rocks of the Rio Doce arc, in response to tectonic activity associated with the exhumation and erosion of thrust fronts, in a scenario consistent with a collisional setting (Fig. 10B).

Together, the major and trace element signatures indicate provenance of protoliths from magmatic arcs and active continental margins, which can be, indeed, of any age older than the regional metamorphism shown by the Salinas rocks (i.e., older than c. $570 \mathrm{Ma}$, cf. Peixoto et al. 2018). In the regional scenario of the Araçuaí orogen, possible candidates to provide sediment sources are the Ediacaran Rio Doce arc (Gonçalves et al. 2016, Tedeschi et al. 2016) and the Rhyacian-Orosirian Mantiqueira arc (Noce et al. 2007), as well as related active margins. Sources in island arcs can be found not only in the juvenile basement of Rio Doce arc, like Rhyacian-Orosirian Juiz de Fora and Pocrane magmatic arcs (Noce et al. 2007, Heilbron et al. 2010, Degler et al. 2018), but also in the basement of the Southern São Francisco craton (Ávila et al. 2010, Teixeira et al. 2015). Although far from the studied region, other possible sources in island arc settings are the juvenile magmatic arcs Serra da Prata and Rio Negro of the Ribeira belt (Tupinambá et al. 2012, Peixoto et al. 2017).

\section{U-Pb (SHRIMP) GEOCHRONOLOGY}

\section{Volcanic clasts from an orthoconglomerate (sample OPU-1995), Salinas Formation type area}

$\mathrm{U}-\mathrm{Pb}$ Concordia diagram for OPU-1995 sample includes 20 zircon grains (Figs. 12 and 13) recovered from a concentrate of pebbles and cobbles of volcanic rocks from a clast-supported metaconglomerate (an orthoconglomerate) cropping out to the South of Salinas town (Fig. 1). The zircons grains include prismatic, euhedral to subhedral crystals. Their size varies from 90 and $200 \mu \mathrm{m}$ in length, and the length/width ratios range from 2:1 to 3:1. They show well-developed oscillatory zoning in CL images, suggesting that the grains were derived from intermediate to felsic igneous rocks (Fig. 12). All the spot analyses have $\mathrm{Th} / \mathrm{U}$ ratios higher than 0.1 , which also indicate igneous growth. However, 11 of the analyses fulfilled the requirements

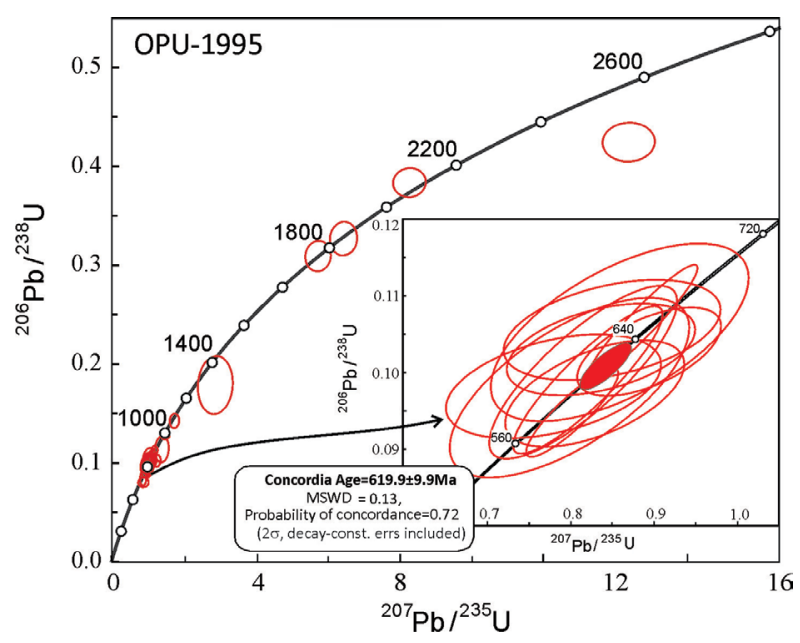

Figure 13. Corcordia diagram showing ages for the pebble-cobble and matrix of sample OPU-1995. Insets showing the Concordia age of $620 \pm 10 \mathrm{Ma}$, yielded by eleven spots.
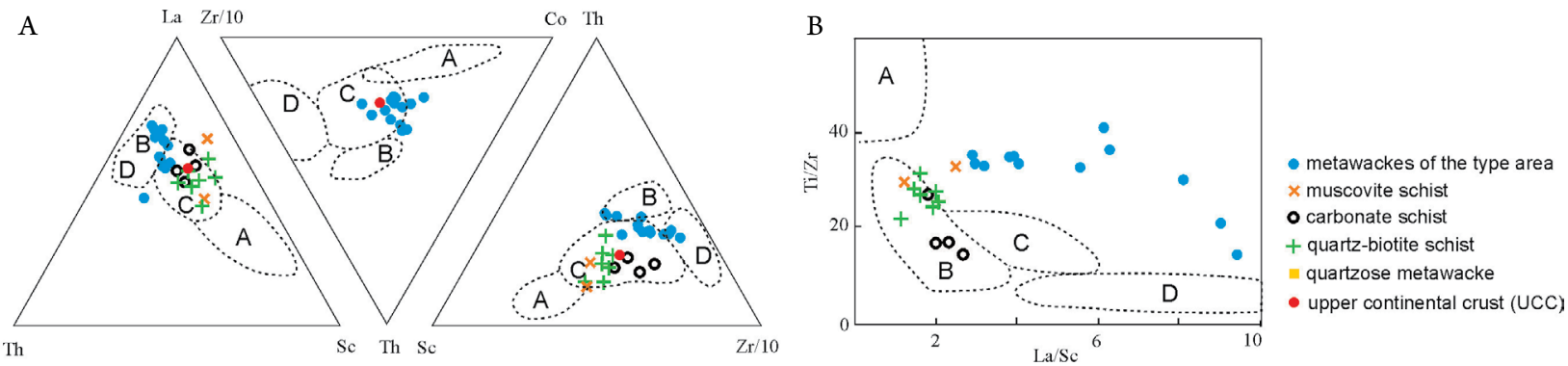

Figure 11. Trace element diagrams for provenance discrimination of protoliths of Salinas rocks: Bhatia \& Crook (1986): (a) Th-La-Sc, $\mathrm{Zr} / 10-\mathrm{Th}-\mathrm{Co}$ and Sc-Th-Zr/10 ternary diagrams; (b) Ti/Zr versus La/Sc. Fields: A, oceanic island arc; B, continental magmatic arc; C, active continental margin; D, passive continental margin.
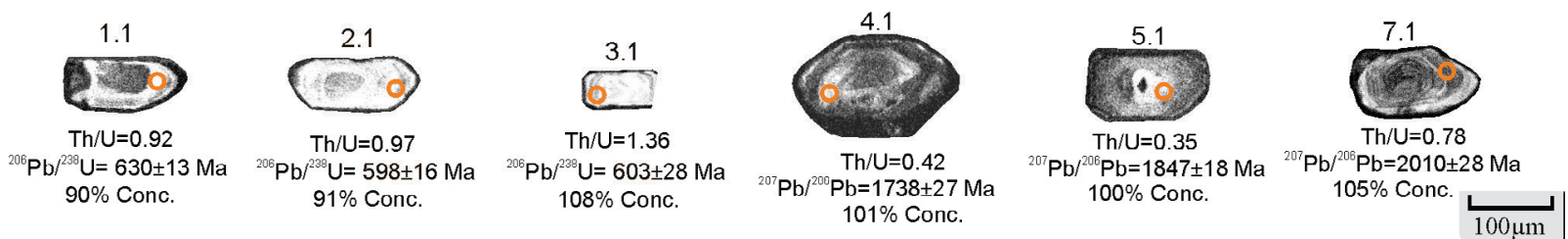

Figure 12. Cathodoluminescence (CL) images showing U-Pb spots in representative zircon grains of sample OPU-1995. 
of Ludwig (2003) Isoplot program to constitute a Concordia age of $620 \pm 10 \mathrm{Ma}$ (Fig. 13). Even though the zircon crystals may derive from different rocks, we consider that they could well be part of the same regional volcanic episode.

Some other grains reported in Table 4, such as zircons $10.1\left({ }^{207} \mathrm{~Pb} /{ }^{206} \mathrm{~Pb}\right.$ age of $\left.2700 \mathrm{Ma}\right), 7.1(2100 \mathrm{Ma}$ age $)$ and 13.1 (720 Ma age), among others, may be considered possible inherited grains within the volcanic rocks, or part of the detrital component of the matrix. Their sources shall be sought either within the nearby continental basement (e.g., the Guanhães or Porteirinha blocks), or within magmatic rocks formed during the early tectonic evolution of Araçuaí orogen. The age of $620 \mathrm{Ma}$ represents an important Ediacaran volcanic episode that contributed for the sedimentary filling of the Salinas basin.

\section{Metawacke (sample SP-54), Salinas Formation type area}

Lima et al. (2002), reporting on the sedimentary deposition within the Salinas synclinorium, presented 15 U-Pb SHRIMP age measurements made on 13 detrital zircon grains, taken from sample SP-54, a wacke typical of the Salinas Formation type area located at about $25 \mathrm{~km} \mathrm{SW}$ from outcrop, where OPU-1995 was collected. The measurements were made by the UGC (Australian National University at Canberra) in 1998, using its SHRIMP instrument. The analytical data are reproduced in Table 5 (Lima et al. 2002). The zircon crystals of SP-54 sample were rounded, exhibited oscillatory zoning in the CL images and presented normal $\mathrm{U}$ content, as well as normal Th/ $\mathrm{U}$ content (0.24-1.22), which are typical of magmatic crystallization. By observing Table 5, only grain six (measured twice) yielded a Paleoproterozoic age. All the other zircons presented Cryogenian to Early Cambrian ages between 700 and $540 \mathrm{Ma}$. This age span is broadly consistent with the age measurement of $620 \pm 10$ Ma made on sample OPU-1995 in this work. Figure 14 includes, along the pertinent part of the Concordia diagram, the analytical points $\left({ }^{207} \mathrm{~Pb} /{ }^{206} \mathrm{~Pb}\right.$ ages $)$ of the dated SP-54 zircons, besides the position of the volcanic episode established from the dating of OPU-1995 sample.

\section{DISCUSSION AND CONCLUSION}

The extraordinary preservation degree of many outcrops makes the Salinas Formation type area an exceptional locality for unraveling the relationships between clastic composition and source rocks information. The presented lithochemical data from Salinas regional rocks and type area ones show a low chemical index of alteration $(\mathrm{CIA}=47$ to 70$)$, and low $\mathrm{SiO}_{2} /$ $\mathrm{Al}_{2} \mathrm{O}_{3}$ ratio (2-8), indicating limited weathering of the sediment source rocks. The composition and immaturity of the metawackes may suggest relatively nearby sources, although this is strongly dependent on sediment transportation velocity and climate conditions. The petrographic characteristics of the studied rocks are consistent with their derivation from

Table 4. U-Pb SHRIMP zircon data from OPU-1995 sample (volcanic pebbles and cobbles from clast-supported conglomerate of the Salinas Formation). Spots that yielded the Concordia age of $620 \pm 10 \mathrm{Ma}$ (Fig. 13) are in bold.

\begin{tabular}{|c|c|c|c|c|c|c|c|c|c|c|c|c|c|c|c|c|c|c|c|c|c|c|}
\hline spot & $\mathbf{U}$ & Th & $T h / U$ & $\mathbf{P} \mathbf{b}_{\mathrm{rad}}$ & ${ }^{204} \mathrm{~Pb}$ & ${ }^{206} \mathbf{P} \mathbf{b}_{\text {com }}$ & $\begin{array}{c}{ }^{238} \mathrm{U} / \\
{ }^{206} \mathrm{~Pb}\end{array}$ & error & $\frac{{ }^{207} \mathrm{~Pb} /}{{ }^{206} \mathrm{~Pb}}$ & error & $\frac{{ }^{207} \mathbf{P b} /}{{ }^{235} \mathbf{U}}$ & - error & $\frac{{ }^{206} \mathbf{P b} /}{{ }^{238} \mathbf{U}}$ & - error & $\begin{array}{l}\text { error } \\
\text { corr. }\end{array}$ & $\begin{array}{r}{ }^{207} \mathbf{P b} / \\
{ }^{206} \mathbf{P b}\end{array}$ & error & $\frac{{ }^{206} \mathbf{P b} /}{{ }^{238} \mathrm{U}}$ & error & $\frac{{ }^{207} \mathbf{P b} /}{{ }^{235} \mathbf{U}}$ & error & Conc. \\
\hline & ppm & ppm & & ppm & ppb & $\%$ & & $1 \sigma$ & & $1 \sigma$ & & $1 \sigma$ & & $1 \sigma$ & & $\begin{array}{l}\text { age } \\
\text { Ma }\end{array}$ & $1 \sigma$ & $\begin{array}{l}\text { age } \\
\text { Ma }\end{array}$ & $1 \sigma$ & $\begin{array}{l}\text { age } \\
\text { Ma }\end{array}$ & $1 \sigma$ & $\%$ \\
\hline 1.1 & 185 & 172 & 0.92 & 22 & 1 & 0.08 & 9.73 & 0.22 & 0.0627 & 0.002 & 0.895 & 0.035 & 0.1027 & 0.0024 & 0.58 & 698 & 65 & 630 & 13 & 645 & 19 & 90 \\
\hline 2.1 & 305 & 297 & 0.97 & 35 & 9 & 0.47 & 10.28 & 0.3 & 0.0614 & 0.0027 & 0.83 & 0.044 & 0.0973 & 0.0028 & 0.55 & 654 & 91 & 598 & 16 & 610 & 25 & 91 \\
\hline 3.1 & 151 & 206 & 1.36 & 19 & 3 & 0.29 & 10.2 & 0.5 & 0.0587 & 70.0032 & 0.799 & 0.058 & 0.098 & 0.0048 & 0.67 & 555 & 116 & 603 & 28 & 593 & 34 & 108 \\
\hline 4.1 & 149 & 64 & 0.42 & 50 & 2 & 0.07 & 3.19 & 0.06 & 0.1064 & 0.0016 & 4.633 & 0.117 & 0.3136 & 0.0064 & 0.8 & 1738 & 27 & 1758 & 31 & 1749 & 22 & 101 \\
\hline 5.1 & 874 & 307 & 0.35 & 302 & 4 & 0.02 & 3.02 & 0.06 & 0.113 & 0.0012 & 5.202 & 0.124 & 0.3315 & 0.0071 & 0.89 & 1847 & 18 & 1845 & 34 & 1846 & 21 & 100 \\
\hline 6.1 & 560 & 73 & 0.13 & 100 & nd & nd & 5.52 & 0.37 & 0.0925 & 0.0015 & 2.329 & 0.161 & 0.1813 & 0.0122 & 0.97 & 1483 & 30 & 1074 & 66 & 1218 & 49 & 73 \\
\hline 7.1 & 119 & 94 & 0.78 & 54 & 1 & 0.03 & 2.57 & 0.04 & 0.1237 & 0.002 & 6.682 & 0.15 & 0.389 & 0.0061 & 0.69 & 2010 & 28 & 2118 & 28 & 2063 & 20 & 105 \\
\hline 8.1 & 114 & 80 & 0.7 & 13 & 6 & 0.85 & 9.47 & 0.24 & 0.058 & 0.0033 & 0.85 & 0.053 & 0.1056 & 0.0027 & 0.41 & 530 & 118 & 648 & 15 & 622 & 29 & 122 \\
\hline 9.1 & 442 & 110 & 0.24 & 35 & 50 & 2.62 & 12.26 & 0.31 & 0.0633 & 0.0039 & 0.717 & 0.048 & 0.0816 & 0.0021 & 0.38 & 718 & 126 & 505 & 12 & 545 & 29 & 70 \\
\hline 10.1 & 267 & 106 & 0.39 & 130 & 3 & 0.04 & 2.32 & 0.04 & 0.194 & 0.0016 & 11.6 & 0.239 & 0.4304 & 0.0081 & 0.92 & 2776 & 13 & 2307 & 36 & 2565 & 19 & 83 \\
\hline 11.1 & 116 & 130 & 1.12 & 15 & 3 & 0.37 & 9.5 & 0.43 & 0.0588 & 80.0034 & 0.859 & 0.063 & 0.1052 & 0.0047 & 0.61 & 557 & 122 & 645 & 27 & 626 & 35 & 116 \\
\hline 12.1 & 84 & 67 & 0.79 & 9 & 4 & 0.82 & 10.18 & 0.29 & 0.0572 & 20.0035 & 0.78 & 0.053 & 0.0982 & 0.0028 & 0.42 & 498 & 129 & 604 & 16 & 582 & 30 & 121 \\
\hline 13.1 & 470 & 376 & 0.8 & 60 & 5 & 0.15 & 8.75 & 0.16 & 0.0611 & 0.0014 & 0.97 & 0.028 & 0.1143 & 0.002 & 0.61 & 640 & 48 & 697 & 11 & 684 & 15 & 109 \\
\hline 14.1 & 1374 & 240 & 0.17 & 140 & 4 & 0.05 & 9.38 & 0.27 & 0.0599 & 9.0006 & 60.887 & 0.027 & 0.1066 & 0.003 & 0.94 & 598 & 22 & 652 & 17 & 640 & 14 & 109 \\
\hline 14.2 & 666 & 87 & 0.13 & 61 & 2 & 0.06 & 10.51 & 0.26 & 0.0614 & 0.0006 & 0.803 & 0.023 & 0.0948 & 0.0024 & 0.88 & 646 & 26 & 587 & 3 & 599 & 6 & 89 \\
\hline 15.1 & 337 & 312 & 0.92 & 39 & 3 & 0.14 & 10.1 & 0.4 & 0.0597 & 0.0012 & 0.821 & 0.036 & 0.0991 & 0.0039 & 0.89 & 591 & 42 & 608 & 23 & 605 & 21 & 103 \\
\hline 15.2 & 465 & 396 & 0.85 & 47 & nd & nd & 11.64 & 0.42 & 0.0602 & 0.0008 & 0.713 & 0.029 & 0.0859 & 0.0031 & 0.9 & 609 & 40 & 533 & 3 & 548 & 8 & 85 \\
\hline 16.1 & 155 & 149 & 0.96 & 19 & 4 & 0.39 & 9.71 & 0.23 & 0.0585 & 0.0029 & 0.837 & 0.046 & 0.103 & 0.0024 & 0.43 & 548 & 105 & 632 & 14 & 614 & 26 & 115 \\
\hline 16.2 & 143 & 153 & 1.06 & 17 & 1 & 0.11 & 16.94 & 0.46 & 0.0591 & 0.0016 & 0.816 & 0.046 & 0.1001 & 0.0046 & 0.82 & 400 & 219 & 603 & 75 & 562 & 74 & 107 \\
\hline 17.1 & 622 & 125 & 0.2 & 64 & 3 & 0.09 & 9.63 & 0.24 & 0.0714 & 0.0013 & 1.023 & 0.033 & 0.1039 & 0.0026 & 0.77 & 933 & 37 & 642 & 5 & 710 & 11 & 66 \\
\hline 18.1 & 136 & 68 & 0.5 & 19 & 2 & 0.19 & 7.71 & 0.09 & 0.0673 & 0.0014 & 1.203 & 0.041 & 0.1297 & 0.0016 & 0.36 & 814 & 64 & 792 & 13 & 798 & 21 & 93 \\
\hline 19.1 & 169 & 192 & 1.13 & 31 & nd & nd & 6.91 & 0.14 & 0.0709 & 0.0015 & 1.413 & 0.044 & 0.1446 & 0.003 & 0.67 & 948 & 52 & 876 & 15 & 897 & 20 & 91 \\
\hline 20.1 & 582 & 633 & 1.08 & 80 & 46 & 1.06 & 8.65 & 0.41 & 0.0709 & 0.0017 & 1.130 & 0.063 & 0.1156 & 0.0055 & 0.85 & 850 & 45 & 702 & 5 & 738 & 13 & 74 \\
\hline
\end{tabular}


mixed sources, especially well recorded by the great variability of clasts contained in the Salinas orthoconglomerate.

The first $\mathrm{U}-\mathrm{Pb}$ geochronological study on clasts of volcanic rocks from a Salinas orthoconglomerate yielded a Concordia age of $620 \pm 10 \mathrm{Ma}$, which strongly suggests a primary provenance from Rio Doce magmatic arc (630-580 Ma; cf. Tedeschi et al. 2016). Furthermore, the U-Pb results given by detrital zircon grains from a Salinas metawacke also show preponderance of ages in the Early Ediacaran.

Robust detrital zircon U-Pb data of Peixoto et al. (2015) provided the youngest main peak at $600 \pm 16 \mathrm{Ma}$ (29\% of the population, corresponding to 31 grains) and a concordant age of $579 \pm 11 \mathrm{Ma}$, which constrain the maximum depositional age for Salinas Formation. This age is identical with the youngest apparent age ( $579 \pm 17 \mathrm{Ma}$, conc. 93\%) obtained from SP-54 sample. $\mathrm{U}-\mathrm{Pb}$ data set indicates a maximum depositional age quite younger than the crystallization of the studied clasts of volcanic rocks and suggests that Salinas basin was mainly filled during the development of Rio Doce magmatic arc.

Overall, the presented ages for Salinas Formation compared with zircon data from other Neoproterozoic rock assemblages suggest sources of sediments relatively close to the Salinas basin, as well as sources located far from it, as the following:

- The Cryogenian South Bahia alkaline province (Rosa et al. 2007), the Tonian to Cryogenian Rio Negro - Serra da Prata magmatic arc and related secondary sources ( $c f$. Degler et al. 2017), and the Late Cryogenian Ribeirão da Folha ophiolite complex (Queiroga et al. 2007, Queiroga 2010). Cryogenian zircon grains could also be derived from the West Congo belt, mainly from the volcanic rocks of La Louila Formation (Thiéblemont et al. 2011) and the Lower Diamictite Formation (Straathof 2011). Potential secondary sources located in the Araçuaí orogen are Macaúbas Group (Kuchenbecker et al. 2015, SantosJunior et al. 2017) and paragneiss complexes (Richter et al. 2016, Degler et al. 2017).
- The Ediacaran Rio Doce magmatic arc (630-580 Ma; Gonçalves et al. 2016, Tedeschi et al. 2016), the collisional G2 super-suite (585-545 Ma; Gradim et al. 2014, Melo et al. 2017a, 2017b), and secondary sources like paragneiss complexes (Richter et al. 2016, Degler et al. 2017) and Rio Doce Group (Novo et al. 2018).

Although chemical-based discrimination diagrams of tectonic settings for provenance studies have been criticized by some authors (Weltje 2006, 2012, Caja et al.2007, Borges et al. 2008), they may be useful if coupled with detailed petrographic studies, confident geochemical scrutiny, and robust geochronological data. In those diagrams (Figs. 10 and 11), most studied samples plot in the continental arc field and extend into the continental active margin field, which are clearly in agreement with interpretations suggested in literature, based on other evidence (Lima et al.2002, Pedrosa-Soares et al. 2008, Peixoto et al. 2015, 2018, Costa et al. 2018). Furthermore, the tectonic signature shown by the discrimination diagrams supports a series of correlations with the available U-Pb geochronological data.

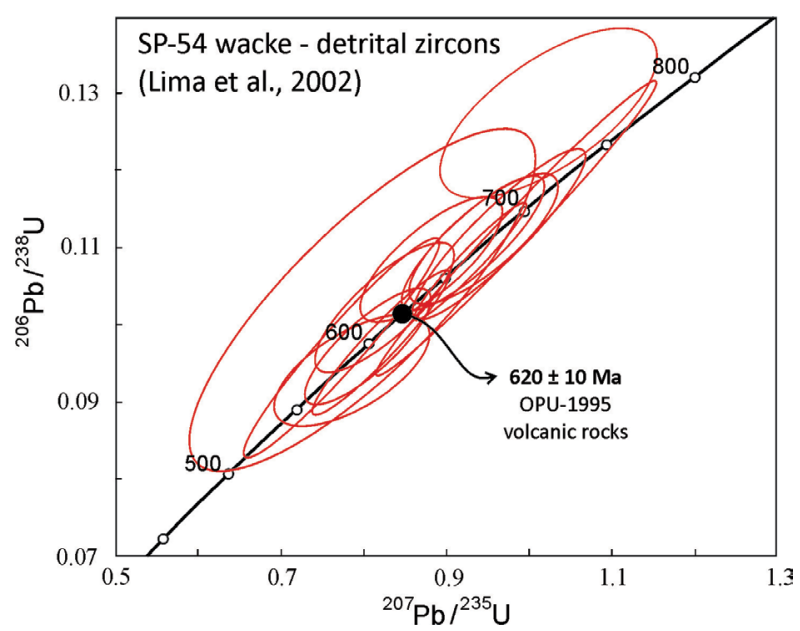

Figure 14. Concordia diagram for the Salinas type area metawacke (sample SP-54; data from Lima et al. 2002).

Table 5. U-Pb SHRIMP zircon data from SP-54 sample (metawacke of the Salinas Formation).

\begin{tabular}{|c|c|c|c|c|c|c|c|c|c|c|c|c|c|c|c|c|c|c|c|c|c|c|}
\hline \multirow{2}{*}{ Spot } & $\mathbf{U}$ & Th & \multirow{2}{*}{$\mathbf{T h} / \mathbf{U}$} & \multirow{2}{*}{$\frac{\mathbf{P \mathbf { b } _ { \text { rad } }}}{\mathbf{p p m}}$} & \multirow{2}{*}{$\begin{array}{l}{ }^{204} \mathrm{~Pb} \\
\text { ppb }\end{array}$} & \multirow{2}{*}{$\begin{array}{c}{ }^{206} \mathbf{P b} \\
\%\end{array}$} & \multirow{2}{*}{$\frac{{ }^{238} \mathrm{U} /}{{ }^{206} \mathrm{~Pb}}$} & \multirow{2}{*}{$\begin{array}{c}\text { error } \\
1 \sigma\end{array}$} & \multirow{2}{*}{$\begin{array}{c}{ }^{207} \mathbf{P b} / \\
{ }^{206} \mathbf{P b}\end{array}$} & \multirow{2}{*}{$\begin{array}{c}\text { error } \\
1 \sigma\end{array}$} & \multirow{2}{*}{$\begin{array}{l}{ }^{207} \mathbf{P b} / \\
{ }^{235} \mathbf{U}\end{array}$} & \multirow{2}{*}{$\begin{array}{c}\text { error } \\
1 \sigma\end{array}$} & \multirow{2}{*}{$\begin{array}{c}{ }^{206} \mathbf{P b} / \\
{ }^{238} \mathbf{U}\end{array}$} & \multirow{2}{*}{$\begin{array}{c}\text { error } \\
1 \sigma\end{array}$} & \multirow{2}{*}{$\begin{array}{l}\text { error } \\
\text { corr. }\end{array}$} & \multirow{2}{*}{$\begin{array}{c}{ }^{207} \mathbf{P b} / / /{ }^{206} \mathbf{P b} \\
\text { age }(\mathrm{Ma})\end{array}$} & \multirow{2}{*}{$\begin{array}{c}\text { error } \\
1 \sigma\end{array}$} & \multirow{2}{*}{$\begin{array}{r}{ }^{206} \mathbf{P b} /{ }^{238} \mathrm{U} \\
\text { age }(\mathrm{Ma})\end{array}$} & \multirow{2}{*}{$\begin{array}{c}\text { error } \\
1 \sigma\end{array}$} & \multirow{2}{*}{$\begin{array}{r}{ }^{207} \mathbf{P b} /{ }^{235} \mathbf{U} \\
\text { age }(\mathrm{Ma})\end{array}$} & \multirow{2}{*}{$\begin{array}{c}\text { error } \\
1 \sigma\end{array}$} & \multirow{2}{*}{$\begin{array}{r}\text { Conc } \\
(\%)\end{array}$} \\
\hline & ppm & ppm & & & & & & & & & & & & & & & & & & & & \\
\hline 1.1 & 176 & 210 & 1.2 & 20 & 3 & 0.28 & 10.63 & 0.33 & 0.0605 & 0.002 & 0.785 & 0.038 & 0.094 & 0.003 & 0.64 & 623 & 74 & 579 & 17 & 588 & 22 & 93 \\
\hline 2.1 & 317 & 299 & 0.94 & 41 & 1 & 0.04 & 9.04 & 0.29 & 0.0611 & 0.001 & 0.931 & 0.035 & 0.1106 & 0.0036 & 0.85 & 642 & 36 & 676 & 21 & 668 & 19 & 105 \\
\hline 3.1 & 240 & 171 & 0.71 & 29 & 8 & 0.51 & 9 & 0.28 & 0.0616 & 0.0012 & 0.944 & 0.037 & 0.1111 & 0.0035 & 0.8 & 661 & 43 & 679 & 20 & 675 & 19 & 103 \\
\hline 4.1 & 324 & 68 & 0.21 & 40 & 33 & 1.51 & 7.85 & 0.28 & 0.0582 & 0.002 & 1.023 & 0.054 & 0.1274 & 0.0045 & 0.67 & 537 & 77 & 773 & 26 & 715 & 27 & 144 \\
\hline 5.1 & 195 & 117 & 0.6 & 21 & 7 & 0.61 & 9.22 & 0.28 & 0.0589 & 0.0013 & 0.881 & 0.035 & 0.1085 & 0.0033 & 0.77 & 564 & 49 & 664 & 19 & 641 & 19 & 118 \\
\hline 6.1 & 238 & 119 & 0.5 & 89 & 2 & 0.04 & 2.95 & 0.09 & 0.1259 & 0.0009 & 5.891 & 0.192 & 0.3393 & 0.0106 & 0.95 & 2042 & 12 & 1883 & 51 & 1960 & 29 & 92 \\
\hline 6.2 & 159 & 116 & 0.73 & 62 & 3 & 0.09 & 2.96 & 0.18 & 0.1416 & 0.0026 & 6.603 & 0.428 & 0.3383 & 0.0203 & 0.92 & 2246 & 32 & 1879 & 98 & 2060 & 59 & 84 \\
\hline 7.1 & 235 & 136 & 0.58 & 28 & 2 & 0.13 & 8.95 & 0.36 & 0.0625 & 0.0009 & 0.963 & 0.043 & 0.1117 & 0.0045 & 0.91 & 685 & 22 & 668 & 29 & 683 & 26 & 99 \\
\hline 8.1 & 840 & 203 & 0.24 & 89 & 4 & 0.08 & 9.24 & 0.27 & 0.0622 & 0.0003 & 0.928 & 0.028 & 0.1082 & 0.0031 & 0.96 & 667 & 15 & 650 & 33 & 662 & 18 & 97 \\
\hline 9.1 & 437 & 272 & 0.62 & 53 & n.d. & n.d. & 8.89 & 0.62 & 0.0634 & 0.0003 & 0.984 & 0.069 & 0.1125 & 0.0078 & 0.99 & 696 & 36 & 681 & 52 & 687 & 46 & 95 \\
\hline 10.1 & 171 & 207 & 1.22 & 21 & 2 & 0.17 & 10.29 & 0.33 & 0.0601 & 0.0011 & 0.804 & 0.031 & 0.0971 & 0.0031 & 0.82 & 599 & 18 & 599 & 25 & 598 & 18 & 99 \\
\hline 11.1 & 207 & 141 & 0.68 & 23 & 5 & 0.4 & 9.78 & 0.33 & 0.0585 & 0.0012 & 0.824 & 0.034 & 0.1022 & 0.0034 & 0.81 & 611 & 19 & 600 & 23 & 627 & 20 & 114 \\
\hline 12.1 & 359 & 276 & 0.77 & 39 & 5 & 0.24 & 10.31 & 0.06 & 0.0578 & 0.0007 & 0.773 & 0.048 & 0.097 & 0.0058 & 0.96 & 581 & 28 & 568 & 37 & 597 & 34 & 115 \\
\hline 12.2 & 316 & 221 & 0.7 & 34 & 1 & 0.05 & 10.24 & 0.4 & 0.0609 & 0.0006 & 0.82 & 0.034 & 0.0976 & 0.0038 & 0.95 & 608 & 19 & 593 & 25 & 601 & 23 & 94 \\
\hline 13.1 & 137 & 177 & 1.29 & 17 & 4 & 0.43 & 9.69 & 0.85 & 0.0561 & 0.0029 & 0.798 & 0.085 & 0.1032 & 0.0091 & 0.82 & 596 & 49 & 592 & 62 & 633 & 53 & 139 \\
\hline
\end{tabular}


According to sedimentological and stratigraphic studies published by Martins-Neto et al. (2001), Lima et al. (2002), Santos et al. (2009) and Costa et al. (2018), the Salinas Formation in its type area records upwards-coarsening turbiditic sedimentation (graded wacke deposits with clast-supported conglomerate lenses at the top), followed by upwards-finning deposits (from wackes to pelites). All these deposits filled that part of the basin with sedimentation coming from NNE, under intermittent but strong seismic activity during the orogenic stages of Araçuaí orogen (Martins-Neto et al. 2001, Lima et al. 2002, Santos et al. 2009, Costa et al. 2018). The presented field, petrographic and lithochemical data allow us to correlate the sedimentary succession of the Salinas type area with the rock assemblage (of quartzose metawacke, carbonate schist, quartz-biotite schist, muscovite schist and sparse lenses of clast-supported metaconglomerate) found in Minas Novas corridor, which is a prolongation of the Salinas synclinorium to the South (Figs. 1 and 4). The clast-supported metaconglomerate lenses found in Minas Novas corridor also contain pebbles and cobbles of felsic to intermediate volcanic rocks (Pedrosa-Soares 1995), which are similar to those found in the Salinas type area (Fig. 3). Although it lacks geochronological data for those clasts, the distribution of orthoconglomerate lenses and regional lithofacies (see map from Pedrosa-Soares 1995) suggests that the Salinas basin was also filled from East in relation to the Minas Novas corridor (Fig. 1), i.e. with sediments provided by Rio Doce arc and collisional granites. Therefore, filling of the Salinas basin seems to have started with pre-collisional flysch-type sediments (Santos et al. 2009, Peixoto et al. 2015), mainly provided by Rio Doce arc, but it would have continued during the collisional stage of Araçuaí orogen.

\section{ACKNOWLEDGEMENTS}

We are grateful for the financial support provided by Brazilian research and development agencies (CNPq, CAPES, CODEMIG, and PETROBRAS). Our gratitude to the scientific and technical staff of the laboratories that provided analytical data for this paper. The authors are very thankful to Claudio Riccomini, Editor-inChief of the Brazilian Journal of Geology, and the anonymous associate editor, and to Robert Pankhurst and an anonymous reviewer for their suggestions, comments, and corrections that greatly help us to improve this manuscript.

\section{ARTICLE INFORMATION}

Manuscript ID: 20190017. Received on: 03/05/2019. Approved on: 05/03/2019.

Author C. D. wrote the first draft of the manuscript and prepared Figures 1 to 12; A. P. S. wrote the abstract and petrography and provided 31 lithochemical analyses, besides improving the manuscript by making corrections and suggestions. Author S. L. provided 14 lithochemical analyses and geochronological data from OPU-1995 sample. Author U. C. got geochronological data and wrote the results of the geochronology section (4.3). Author K. S. provided Figures 13 and 14 and improved the geochronology section.

Competing interests: The authors declare no competing interests.

\section{REFERENCES}

Alkmim F.F., Marshak S., Pedrosa-Soares A.C., Peres G.G., Cruz S.C., Whittington A. 2006. Kinematic evolution of the Araçuaí-West Congo orogen in Brazil and Africa: nutcracker tectonics during the Neoproterozoic assembly of Gondwana. Precambrian Research, 149(1-2):43-64. https:// doi.org/10.1016/j.precamres.2006.06.007

Augustsson C., Bahlburg H. 2008. Provenance of late Palaeozoic metasediments of the Patagonian proto-Pacific margin (southernmost Chile and Argentina). International Journal Earth Science (Geologische Rundschau), 97(1):71-88. https://doi.org/10.1007/s00531-006-0158-7

Ávila C.A., Teixeira W., Cordani U.G., Moura C.A.V., Pereira R.M. 2010. Rhyacian (2.23-2.20 Ga) juvenile accretion in the southern São Francisco craton, Brazil: Geochemical and isotopic evidence from the Serrinha magmatic suite, Mineiro belt. Journal of South American Earth Sciences, 29(2):464-482. https://doi.org/10.1016/j.jsames.2009.07.009

Baars F.J., Grossi-Sad J.H., Fonseca E. 1997. Geologia da Folha Capelinha. In: Grossi-Sad J.H., Lobato L.M., Pedrosa-Soares A.C., Soares-Filho B.S (eds.), Projeto Espinhaço em CD-ROM. Belo Horizonte, COMIG, p. 1373-1503.

Bhatia M.R. 1985. Rare element geochemistry of Australian Paleozoic graywackess and mudrocks: Provenance and tectonic control. Sedimentary Geology, 45(1-2):97-113. https://doi. org/10.1016/0037-0738(85)90025-9

Bhatia M.R., Crook K.A.W. 1986. Trace elements characteristics of graywackess and tectonic setting discriminations of sedimentary basins Contributions to Mineralogical and Petrology, 92(2):181-193. https://doi. org/10.1007/BF00375292

Borges J.B., Huh Y., Moon S., Noh H. 2008. Provenance and weathering control on river bed sediments of the eastern Tibetan Plateau and the Russian Far East. Chemical Geology, 254(1-2):52-72. https://doi.org/10.1016/j. chemgeo.2008.06.002
Boynton W.V. 1984. Cosmochemistry of the rare earth elements; meteorite studies. In: Henderson P. (ed.). Rare earth element geochemistry. Amsterdam, Elsevier Science Publishing Company, p. 63-114.

Caja M.A., Marfil R., Lago M., Salas R., Ramseyer K. 2007. Provenance discrimination of Lower Cretaceous synrift sandstones (eastern Iberian Chain Spain): constraints fromdetrital modes, heavy minerals, and geochemistry. In: Arribas J., Critelli S.,Johnsson M.J. (eds.). Sedimentary Provenance and Petrogenesis: Perspectives from Petrography and Geochemistry. United States, Geological Society of America, v. 420, p. 181-197. https://doi.org/10.1130/SPE420

Costa F.G.D., Alkmim F.F., Magalhães P.M. 2018. The Ediacaran Salinas turbidites, Araçuaí Orogen, MG: tectonics and sedimentation interplay in a syn-orogenic basin. Brazilian Journal of Geology, 48(4):783-804.

Degler R., Pedrosa-Soares A., Dussin I., Queiroga G., Schulz B. 2017. Contrasting provenance and timing of metamorphism from paragneisses of the Araçuaí-Ribeira orogenic system, Brazil: Hints for Western Gondwana assembly. Gondwana Research, 51:30-50. http://dx.doi.org/10.1016/j.gr.2017.07.004

Degler R., Pedrosa-Soares A., Novo T., Tedeschi M., Silva L.C., Dussin L., Lana C. 2018. Rhyacian-Orosirian isotopic records from the basement of the Araçuaí-Ribeira orogenic system (SE Brazil): Links in the Congo-São Francisco palaeocontinent. Precambrian Research, 317:179-195. https:// doi.org/10.1016/j.precamres.2018.08.018

Gonçalves L., Alkmim F.F., Pedrosa-Soares A.C., Dussin I., Valeriano C., Lana C. Tedeschi M. 2016. Granites of the intracontinental termination of a magmatic arc: an example from the Ediacaran Araçuaí orogen, southeastern Brazil Gondwana Research, 36:439-459. https://doi.org/10.1016/j.gr.2015.07.015

Gradim C., Roncato J., Pedrosa-Soares A.C., Cordani U.G., Dussin I.A., Alkmim F.F., Queiroga G., Jacobsohn T., Silva L.C., Babinski M. 2014. The hot back-arc zone of the Araçuaí orogen, Eastern Brazil: from sedimentation to granite generation. Brazilian Journal of Geology, 44(1):155-180. https:// doi.org/10.5327/Z2317-4889201400010012 
Grossi-Sad J.H., Motta E.G.M. 1991. Geologia e prospecção geoquímica na área Capelinha - Turmalina - Minas Novas, MG. Relatório para ACESITA. Belo Horizonte, GEOSOL.

Guimarães M.L.V., Grossi-Sad J.H. 1997. Geologia da Folha de Malacacheta. In: Grossi-Sad J.H., Lobato L.M., Pedrosa-Soares A.C., Soares-Filho B.S (eds.). Projeto Espinhaço em CD-ROM. Belo Horizonte, COMIG, p. 1505-1574.

Heilbron M., Duarte B., Valeriano C., Simonetti A., Machado N., Nogueira J. 2010. Evolution of reworked Paleoproterozoic basement rocks within the Ribeira belt (Neoproterozoic), SE-Brazil, based on $\mathrm{U} \mathrm{Pb}$ geochronology: implications for paleogeographic reconstructions of the São FranciscoCongo paleocontinent. Precambrian Research, 178:136-148.

Herron M.M. 1988. Geochemical classification of terrigenous sands and shales from core or log data. Journal of Sedimentary Petrology, 58(5):820-829. https://doi.org/10.1306/212F8E77-2B24-11D7-8648000102C1865D

Janousek V., Farrow C.M., Erban V. 2006. Interpretation of whole-rock geochemical data in igneous geochemistry: introducing Geochemical Data Toolkit (GCDkit). Journal of Petrology, 47(6):1255-1259. https://doi. org/10.1093/petrology/egl013

Karfunkel J., Pedrosa-Soares A.C., Dossin I.A. 1985. O Grupo Macaúbas em Minas Gerais, revisão dos conhecimentos. In: Simpósio de Geologia, 3., MG. Anais... p. 45-59.

Kroonenberg S.B. 1994. Effects of provenance, sorting and weathering on the geochemistry of fluvial sands from different tectonic and climatic environments. In: International Geological Congress, 29., Kyoto. Proceedings..., p. 69-81.

Kuchenbecker M., Pedrosa-Soares A.C., Babinski M., Fanning M. 2015. Detrital zircon age patterns and provenance assessment for pre-glacial to post-glacial successions of the Neoproterozoic Macaúbas Group, Araçuaí orogen, Brazil. Precambrian Research, 266:12-26. http://dx.doi. org/10.1016/j.precamres.2015.04.016

Lima S.A.A., Martins-Neto M., Pedrosa-Soares A.C., Cordani U.G., Nutman A. 2002. A Formação Salinas na área tipo, NE de Minas Gerais: uma proposta de revisão da estratigrafia da Faixa Araçuaí com base em evidências sedimentares, metamórficas, e idades U-Pb SHRIMP. Revista Brasileira de Geociências, 32(4):491-500.

Ludwig K.R. 2003. Using Isoplot/Ex, version 3.00, a geochronological toolkit for Microsoft Excel. Berkeley, Geochronology Center (Special Publication), $43 \mathrm{p}$

Martins-Neto M.A., Pedrosa-Soares A.C., Lima S.A.A. 2001. Tectonosedimentary evolution of sedimentary basins from Late Paleoproterozoic to Late Neoproterozoic in the São Francisco craton and Araçuaí fold belt, eastern Brazil. Sedimentary Geology, 141:343-370. http://dx.doi. org/10.1016/S0037-0738(01)00082-3

McLennan S.M., Hemming S.R., McDaniel D.K., Hanson G.N. 1993. Geochemical approaches to sedimentation, provenance and tectonics. In: Johnsson M.J., Basu A. (eds.). Processes controlling the composition of clastic sediments. United States, Geological Society of America, v. 284, p. 21-40. https://doi.org/10.1130/SPE284-p21

Melo M.G., Lana C., Stevens G., Pedrosa-Soares A.C., Gerdes A., Alkmin L.A., Nalini H.A., Alkmim F.F. 2017a. Assessing the isotopic evolution of S-type granites of the Carlos Chagas Batholith, SE Brazil: Clues from $\mathrm{U} / \mathrm{Pb}$, Hf isotopes, $\mathrm{Ti}$ geothermometry and trace element composition of zircon. Lithos, 284-285:730-750. http://dx.doi.org/10.1016/j. lithos.2017.05.025

Melo M.G., Stevens G., Lana C., Pedrosa-Soares A.C., Frei D., Alkmim F.F., Alkmin L.A. 2017b. Two cryptic anatectic events within a syn-collisional granitoid from the Araçuaí orogen (southeastern Brazil): Evidence from the polymetamorphic Carlos Chagas batholith. Lithos, 277:51-71. http:// dx.doi.org/10.1016/j.lithos.2016.10.012

Moraes L.J. 1932. Área ocupada pela Formação Macaúbas no norte de Minas Gerais. Anais da Academia Brasileira de Ciências, 4(3):111-114.

Morton A.C., Hallsworth C.R. 1999. Processes controlling the composition of heavy mineral assemblages in sandstones. Sedimentary Geology, 124(14):3-29. https://doi.org/10.1016/S0037-0738(98)00118-3

Nesbitt H.W. 2003. Petrogenesis of siliciclastic sediments and sedimentary rocks. In: Lentzm D.R. (ed.). Geochemistry of Sediments and Sedimentary Rocks: Evolution Considerations to Mineral Deposit-Forming Environments. Sant John's, Geological Association of Canada, v. 4, p. 39-51.
Nesbitt H.W., Young G.M. 1984. Prediction of some weathering trends of plutonic and volcanic rocks based on thermodynamic and kinetic considerations. Geochimica et Cosmochimica Acta, 48(7):1523-1534. https://doi.org/10.1016/0016-7037(84)90408-3

Noce C.M., Pedrosa-Soares A.C., Silva L.C., Armstrong R., Piuzana D. 2007. Evolution of polyciclic basement complexes in the Araçuaí orogen based on $\mathrm{U}-\mathrm{Pb}$ SHRIMP data: Implications for Brazil-Africa links in Paleoproterozoic time. Precambrian Research, 159(1):60-78. http://dx.doi.org/10.1016/j. precamres.2007.06.001

Novo T.A., Pedrosa-Soares A.C., Vieira V.S., Dussin I.A., Silva L.C. 2018. The Rio Doce Group revisited: an Ediacaran arc-related volcanosedimentary basin, Araçuaí orogen (SE Brazil). Journal of South American Earth Sciences, 85:345-361. https://doi.org/10.1016/j. jsames.2018.05.013

Oliveira M.J.R., Mourão M.A., Noce C.M, Grossi-Sad J.H. 1997. Geologia da Folha Grão Mogol. In: Grossi-Sad J.H., Lobato L.M., Pedrosa-Soares A.C., Soares-Filho B.S. (eds.). Projeto Espinhaço em CD-ROM. Belo Horizonte, COMIG, p. 620-677.

Pedrosa-Soares A.C. 1984. Metamorfismo, granitogênese e mineralizações associadas na região de Coronel Murta, NE de Minas Gerais. MS Dissertation, Instituto de Geociências, Universidade de Brasília, Brasília, 236 p.

Pedrosa-Soares A.C., Noce C.M., Monteiro R.L.B.P., Mourão M.A.A., Lima A. J. 1990a. A evolução monocíclica da Faixa Araçuaí no Médio Jequitinhonha: evidências estratigráficas, estruturais e metamórficas. In: 36 Congresso Brasileiro de Geologia, 1990, Natal. Boletim de Resumos. Natal: SBG Núcleo Nordeste, p. 292-292.

Pedrosa-Soares A.C., Monteiro R.L.B.P., Noce C.M., Silva F.F.E., Oliveira M.J.R., Schetino A. 1990b. Caracterização de uma sequência vulcanosedimentar distal na Faixa Araçuaí, MG: bacia oceânica restrita?. In: 36 Congresso Brasileiro de Geologia, 1990, Natal. Boletim de Resumos. Natal: SBG Núcleo Nordeste, p. 308-308.

Pedrosa-Soares A.C. 1995. Potencial Aurífero do Vale do Araçuaí, MG: História da exploração, geologia e controle-tectono metamórfico. PhD Thesis, Instituto de Geociências, Universidade de Brasília, Brasília, 177 p.

Pedrosa-Soares A.C. 1997a. Geologia da Folha Araçuaí. In: Grossi-Sad J.H., Lobato L.M., Pedrosa-Soares A.C., Soares-Filho B.S. (eds.), Projeto Espinhaço em CD-ROM. Belo Horizonte, COMIG, p. 715-852.

Pedrosa-Soares A.C. 1997b. Geologia da Folha Jenipapo. In: Grossi-Sad J.H., Lobato L.M., Pedrosa-Soares A.C., Soares-Filho B.S. (eds.). Projeto Espinhaço em CD-ROM. Belo Horizonte, COMIG, p. 1053-1198.

Pedrosa-Soares A.C., Baars F.J., Lobato L.M., Magni M.C.V., Faria L.F. 1993. Arquitetura tectono-metamórfica do setor central da Faixa Araçuaí e suas relações com o Complexo Guanhães. In: Simpósio Nacional de Estudos Tectônicos, 4., Belo Horizonte. Proceedings..., p. 176-182.

Pedrosa-Soares A.C., Campos C.P., Noce C.M., Silva L.C., Novo T.A., Roncato J., Medeiros S., Castañeda C., Queiroga G.N., Dantas E., Dussin I.A., Alkmim F.F. 2011. Late Neoproterozoic-Cambrian granitic magmatism in the Araçuaí orogen (Brazil), the Eastern Brazilian Pegmatite Province and related mineral resources. Geological Society of London, Special Publications, 350:25-51. https://doi.org/10.1144/SP350.3

Pedrosa-Soares A.C., Grossi-Sad J.H. 1997. Geologia da Folha Minas Novas. In: Grossi-Sad J.H., Lobato L.M., Pedrosa-Soares A.C., SoaresFilho B.S. (eds.). Projeto Espinhaço em CD-ROM. Belo Horizonte, COMIG, p. 952-1052.

Pedrosa-Soares A.C., Leonardos O.H. 1996. O Distrito Aurífero das Minas Novas do Araçuaí: Uma abordagem fundamentada em dados históricos e geológicos. Revista Escola de Minas (REM), 49(4):33-38.

Pedrosa-Soares A.C., Noce C.M., Vidal P., Monteiro R., Leonardos O.H. 1992. Toward a new tectonic model for the Late Proterozoic Araçuaí (SE Brazil)-West Congolian (SW Africa) Belt. Journal of South American Earth Science, 6(1-2):33-47. http://dx.doi. org/10.1016/0895-9811(92)90015-Q

Pedrosa-Soares A.C., Noce C.M., Wiedemann C.M., Pinto C.P. 2001. The Araçuaí-West Congo orogen in Brazil: an overview of a confined orogen formed during Gondwanland assembly. Precambrian Research, 110 (1):307323. https://doi.org/10.1016/S0301-9268(01)00174-7

Pedrosa-Soares A.C., Oliveira M.J.R. 1997. Geologia da Folha Salinas. In: Grossi-Sad J.H., Lobato L.M., Pedrosa-Soares A.C., Soares-Filho B.S. (eds.), Projeto Espinhaço em CD-ROM. Belo Horizonte, COMIG, p. 419-542. 
Pedrosa-Soares A.C., Vidal P., Leonardos O.H., Brito-Neves B.B. 1998. Neoproterozoic oceanic remnants in eastern Brazil: further evidence and refutation of an exclusively ensialic evolution for the Araçuaí-West Congo orogen. Geology, 26(6):519-522. https://doi.org/10.1130/0091-7613(1998)026\%3C0 519:NORIEB\%3E2.3.CO;2

Pedrosa-Soares A.C., Alkmim F.F., Tack L., Noce C.M., Babinski M., Silva L.C., Martins-Neto M. 2008. Similarities and differences between the Brazilian and African counterparts of the Neoproterozoic Araçuaí-West Congo orogen. In: Pankhurst J.R., Trouw R.A.J., Brito Neves B.B., De Wit M.J. (Eds.) West Gondwana: Pre-Cenozoic Correlations across the South Atlantic Region. London, Geological Society of London, Special Publications, 294:153-172.

Peixoto C.A., Heilbron M., Ragatky D., Armstrong R., Dantas E., Valeriano C.M., Simonetti A. 2017. Tectonic evolution of the Juvenile Tonian Serra da Prata magmatic arc in the Ribeira belt, SE Brazil: Implications for early west Gondwana amalgamation. Precambrian Research, 302:221-254. https:// www.researchgate.net/deref/http\%3A\%2F\%2Fdx.doi.org\%2F10.1016\%2Fj. precamres.2017.09.017

Peixoto E., Alkmim F.F., Pedrosa-Soares A., Lana C., Chaves A.O. 2018 Metamorphic record of collision and collapse in the Ediacaran-Cambrian Araçuaí orogen, SE-Brazil: Insights from $P$ - T pseudosections and monazite dating. Journal of Metamorphic Geology, 36(2):147-172. https://doi.org/10.1111/jmg.12287

Peixoto E., Pedrosa-Soares A.C., Alkmim F.F., Dussin I.A. 2015. A suturerelated accretionary wedge formed in the Neoproterozoic Araçuaí orogen (SE Brazil) during Western Gondwanaland assembly. Gondwana Research, 27(2):878-896. https://doi.org/10.1016/j.gr.2013.11.010

Pettijohn F.J., Potter P.E., Siever R. 1972. Sand and Sandstone. New York, Springer-Verlag, $618 \mathrm{p}$.

Queiroga G.N. 2010. Caracterização de restos de litosfera oceânica do Orógeno Aracuaí entre os paralelos $17^{\circ}$ e $21^{\circ} \mathrm{S}$. PhD Thesis, Instituto de Geociências, Universidade Federal de Minas Gerais, Belo Horizonte, 180 p.

Queiroga G.N., Pedrosa-Soares A.C., Noce C.M., Alkmim F.F., Pimentel M.M., Dantas E., Martins M., Castañeda C., Suita M.T.F., Prichard H. 2007. Age of the Ribeirão da Folha ophiolite, Araçuaí Orogen: the U-Pb zircon dating of a plagiogranite. Geonomos, 15(1):61-65.

Richter F., Lana C., Stevens G., Buick I., Pedrosa-Soares A.C., Alkmim F.F., Cutts K. 2016. Sedimentation, metamorphism and granite generation in a back-arc region: Records from Ediacaran Nova Venécia Complex (Araçuaí Orogen, Southeastern Brazil). Precambrian Research, 272:78-100. https:// doi.org/10.1016/j.precamres.2015.10.012

Rosa M.L.S., Conceição H., Macambira M.J., Galarza M.A., Cunha M.P., Menezes R.C.L., Marinho M.M., Cruz-Filho B.E., Rios D.C. 2007. Neoproterozoic anoro-genic magmatism in the Southern Bahia Alkaline Province of NE Brazil: $\mathrm{U}-\mathrm{Pb}$ and $\mathrm{Pb}-\mathrm{Pb}$ ages of the blue sodalite syenites. Lithos, 97(1):88-97. http://dx.doi.org/10.1016/j.lithos.2006.12.011

Rosen O.M. 1992. Graywackes of the Precambrian metamorphic complexes: Composition and paleogeodynamic reconstructions. International Geology Review, 34(12):1169-1186. https://doi.org/10.1080/00206819209465660

Roser B.P., Korsch R.J. 1986. Determination of tectonic setting of sandstone-mudstone suites using $\mathrm{SiO}_{2}$ and $\mathrm{K}_{2} \mathrm{O} / \mathrm{Na}_{2} \mathrm{O}$ ratio. Journal of Geology, 94(5):635-650.

Roser B.P., Korsch R.J. 1999. Geochemical characterization, evolution and source of Mesozoic accretionary wedge: the Torlesse terrane, New Zealand. Geological Magazine, 136:493-512.

Santos R.F, Alkmim F.F., Pedrosa-Soares A.C. 2009. A Formação Salinas, Orógeno Aracuaí (MG): história deformacional e significado tectônico. Revista Brasileira de Geociências, 39(1):81-100.

Santos-Junior A.G., Pedrosa-Soares A.C., Karfunkel J., Caetano-Filho S., Babinski M., Trindade R., Lana C. 2017. Characterization of the Macaúbas and Bambui groups at the Paraúna dan área, Araçuaí Orogen: new geochronological and isotopic data. In: Geosudeste. Proceedings... Diamantina, Sociedade Brasileira de Geologia.
Slack J.F., Höy T. 2000. Geochemistry and provenance of clastic metasedimentary rocks of the Aldridge and Fort Steele Formation, Purcell Supergroup, SE British Columbia. In: Lydon J.W., Höy T., Slack J.F., Knapp M.E. (eds.). The geological environment of the Sullivan Deposit, British Columbia. Canada,Geological Association of Canada, v. 1, p. 180-201.

Straathof G.B. 2011. Neoproterozoic Low Latitude Glaciations: An African Perspective. PhD Thesis, School of GeoSciences, University of Edinburgh, Edinburgh, $251 \mathrm{p}$

Sun Q.,Zhou Y.,Zhao T., Wang W.2017. Geochronology and geochemistry of the Paleoproterozoic Yinyugou Group in the southern North China Craton; implications for provenance and tectonic evolution. Precambrian Research, 296:120-147. http://dx.doi.org/10.1016/j.precamres.2017.04.012

Taylor S.T., McLennan S.M. 1985. The Continental Crust: Its Composition and Evolution. Oxford, Blackwell, $312 \mathrm{p}$.

Tedeschi M., Novo T., Pedrosa-Soares A., Dussin I., Tassinari C., Silva L.C., Gonçalves L., Alkmim F.F., Lana C., Figueiredo C., Dantas E., Medeiros S., Campos C., Corrales F., Heilbron M. 2016. The Ediacaran Rio Doce magmatic arc revisited (Araçuaí-Ribeira orogenyc system, SE Brazil). Journal of South American Earth Sciences, 68:167-186. http://dx.doi.org/10.1016/j. jsames.2015.11.011

Teixeira W., Ávila C.A., Dussin I.A., Corrêa Neto A.V., Bongiolo E.M., Santos J.O.S., Barbosa N.S. 2015. Zircon U-Pb-Hf, Nd-Sr constraints and geochemistry of the Resende Costa Orthogneiss and coeval rocks: new clues for a juvenile accretion episode $(2.36-2.33 \mathrm{Ga})$ in the Mineiro belt and its role to the long-lived Minas accretionary orogeny. Precambrian Research, 256:148-169. https://doi.org/10.1016/j. precamres.2014.11.009

Thiéblemont D., Prian J.P., Goujou J.C., Boulingui B., Ekogha H., Kassadou A.B., Simo-Ndounze S., Walemba A., Préat A., Theunissen K., Cocherie A. Guerrot C. 2011. Timing and characteristics of Neoproterozoic magmatism in SW-Gabon:first geochronogical and geochemical data on the WestCongolian orogen in Gabon (SYSMIN project, Gabon 2005-2009). In: Colloquium of African Geology, 23. Proceedings... Johannesburg: Geological Society of Africa.

Tupinambá M., Heilbron M., Valeriano C.M., Porto Jr. R., Blanco de Dios F., Machado N., Silva L.G.E., Almeida J.C.H. 2012. Juvenile contribution of the Neoproterozoic Rio Negro magmatic arc (Ribeira Belt, Brazil): implications for western Gondwana amalgamation. Gondwana Research, 21:422-438.

Verma S.P., Armstrong-Altrin J.S. 2013. New multi-dimensional diagrams for tectonic discrimination of siliciclastic sediments and their application to Precabrian basins. Chemical Geology, 355:117-133. https://doi. org/10.1016/j.chemgeo.2013.07.014

Weltje G.J. 2006. Ternary sandstone composition and provenance: an evaluation of the "Dickinson model". In: Buccianti A., Mateu-Figueras G., Pawlowsky-Gahn V. (eds.), Compositional Data Analysis in the Geosciences: From Theory to Practice. London, Geological Society of London, Special Publications, v. 264, p. 9-99.

Weltje G.J. 2012. Quantitative models of sediment generation and provenance: state of the art and future developments. Sedimentary Geology, 280:4-20. https://doi.org/10.1016/j.sedgeo.2012.03.010

Williams I.S. 1998. U-Th-Pb geochronology by ion microprobe. In: McKibben M. A., Shanks I., Ridley W. C. P., Ridley W. I. (eds.), Application of Microanalytical Technique to Understanding Mineralizing Process. Reviews in Economic Geology, v. 7, p. 1-35.

Zhang K.J. 2004. Secular geochemical variations of the Lower Cretaceous siliciclastic rocks from central Tibet (China) indicate a tectonic transition from continental collision to back-arc rifting. Earth and Planetary Science Letters, 229(1):73-89. http://dx.doi.org/10.1016/j.epsl.2004.10.030 